Supplement of Earth Surf. Dynam., 7, 475-489, 2019

https://doi.org/10.5194/esurf-7-475-2019-supplement

(c) Author(s) 2019. This work is distributed under

the Creative Commons Attribution 4.0 License.

(c) (1)
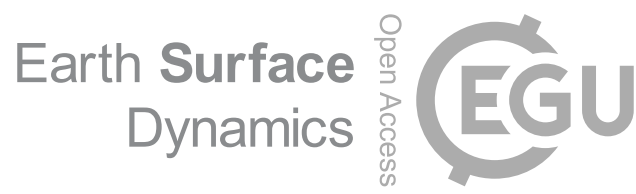

Supplement of

\title{
Determining the optimal grid resolution for topographic analysis on an airborne lidar dataset
}

\section{Taylor Smith et al.}

Correspondence to: Taylor Smith (tasmith@uni-potsdam.de)

The copyright of individual parts of the supplement might differ from the CC BY 4.0 License. 


\section{Data and Methods}

As a second synthetic surface, we generate a sphere with a radius of one, with elevations defined as $z=\sin \left(\arccos \left(\sqrt{x^{2}+y^{2}}\right)\right)$. The following figures contain both the Gaussian hill data and the sphere data.
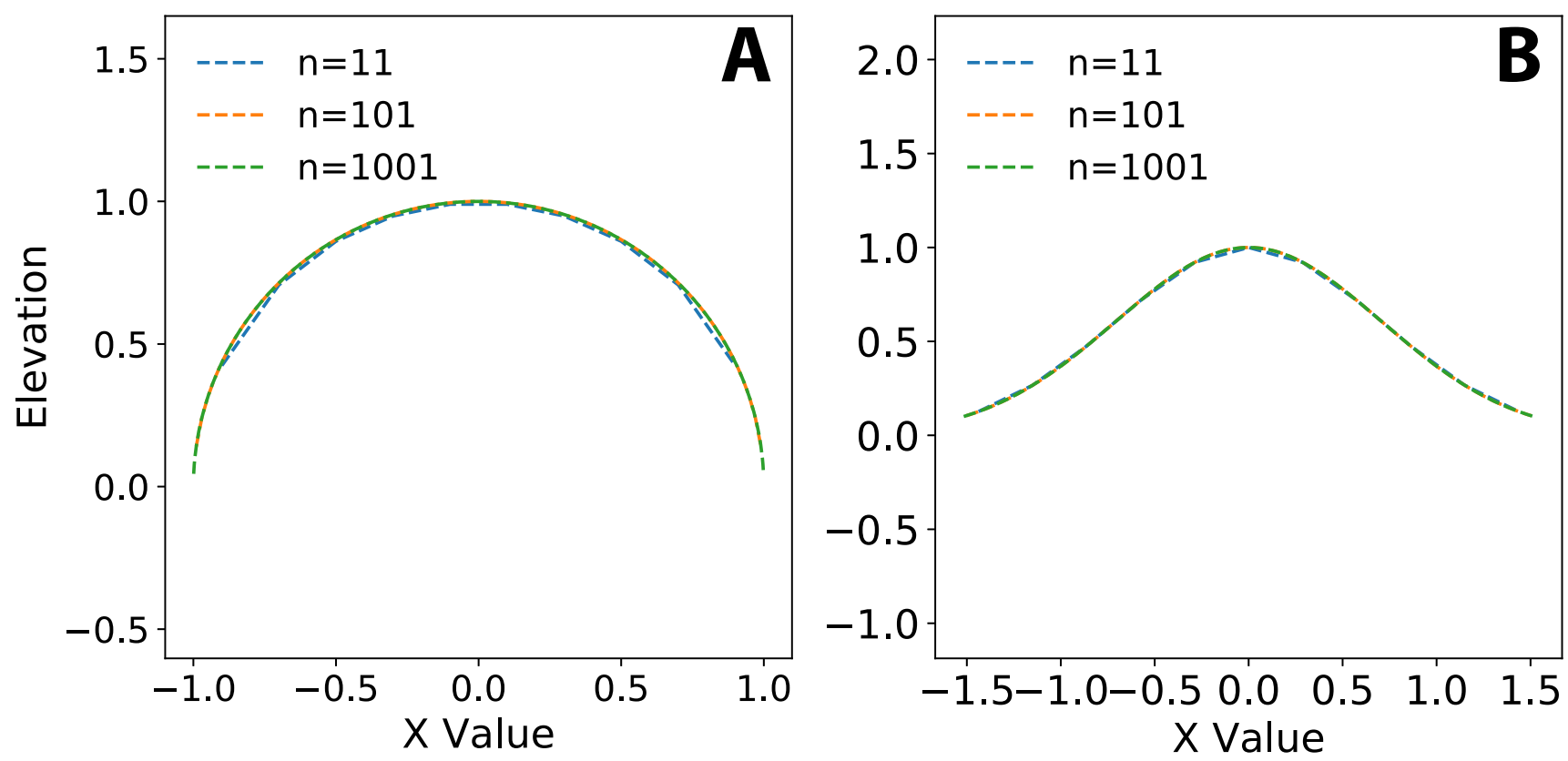

Figure S1. Sphere (left) and Gaussian Hill (right) elevations at $n=11,101$, and 1001 in profile. 

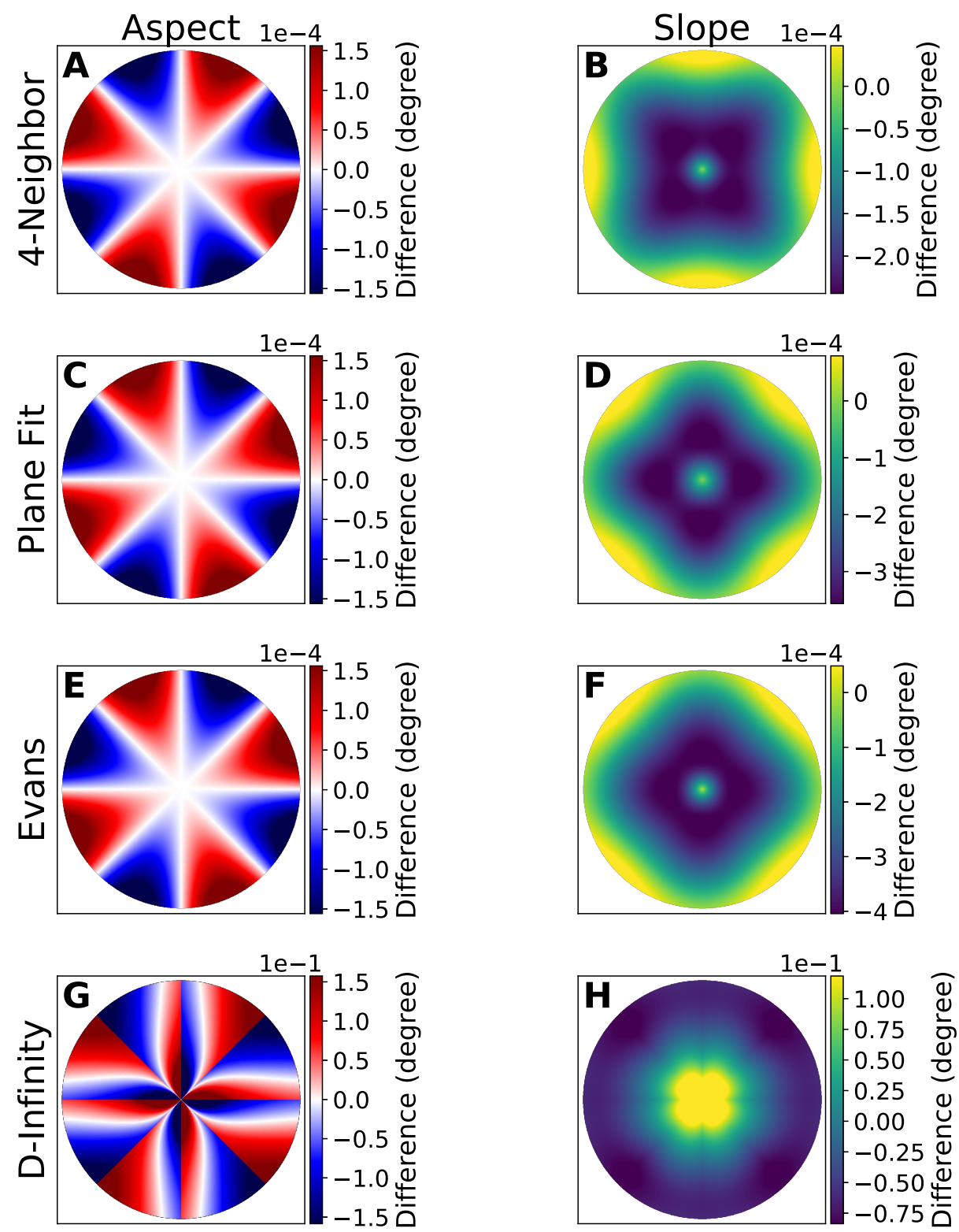

Figure S2. Absolute offset from the analytical solution for slopes and aspects on a Gaussian Hill (n=1001) for (1) 4-neighbor method, (2) plane fits, (3) Evans (Evans, 1980) method, and (4) D-Infinity (Tarboton, 1997). Colors scaled from 5th to 95th percentiles. 

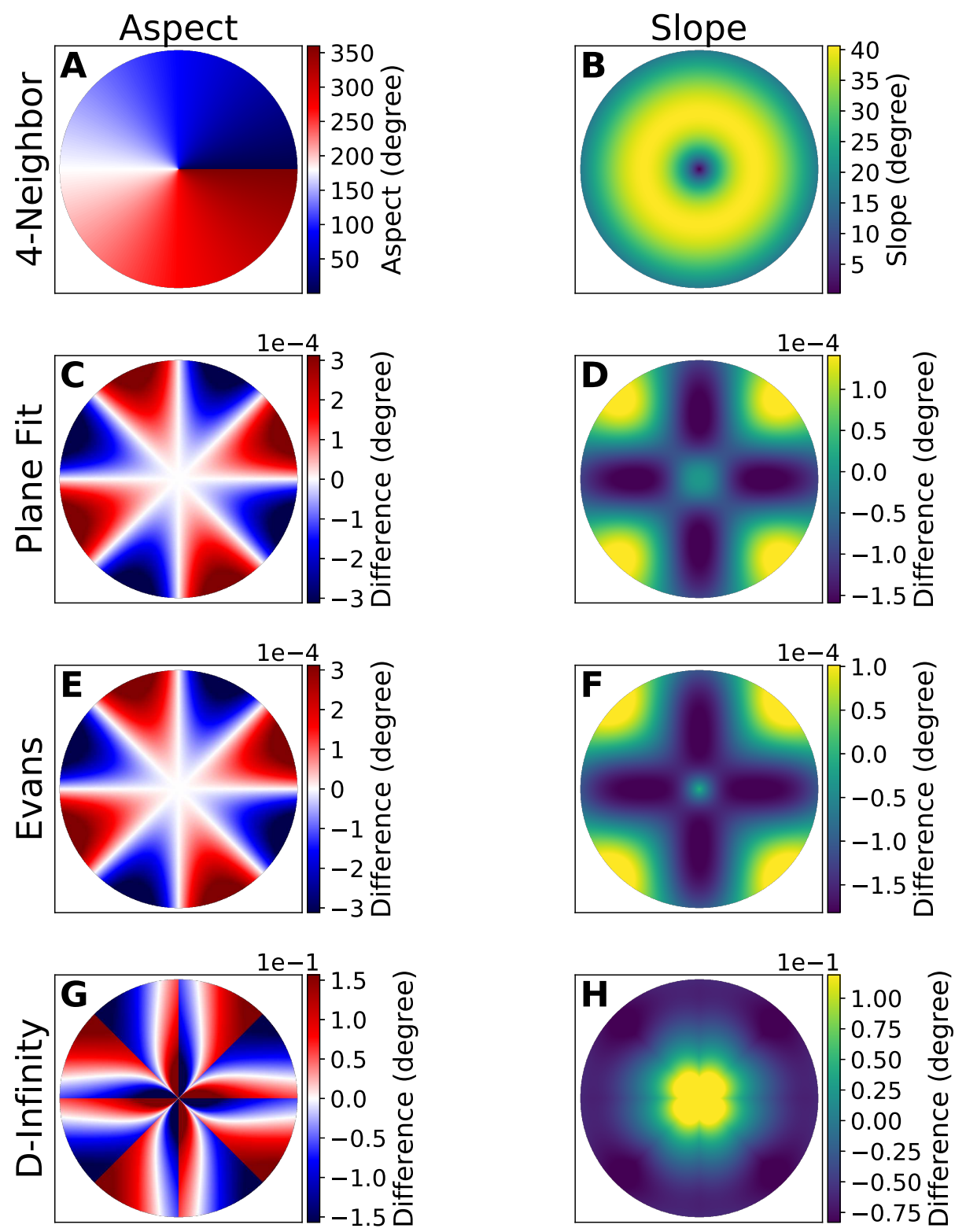

Figure S3. Slope and Aspect for a Gaussian Hill (n=1001) using four different methods. (1) 4-neighbor method, (2) difference between plane fits and 4-neighbor method, (3) difference between Evans (Evans, 1980) method and 4-neighbor method, and (4) difference between D-Infinity (Tarboton, 1997) and 4-neighbor method. Colors scaled from 5th to 95th percentiles. 


\section{Sources of Error in Topographic Metrics}

\subsection{Truncation Error}
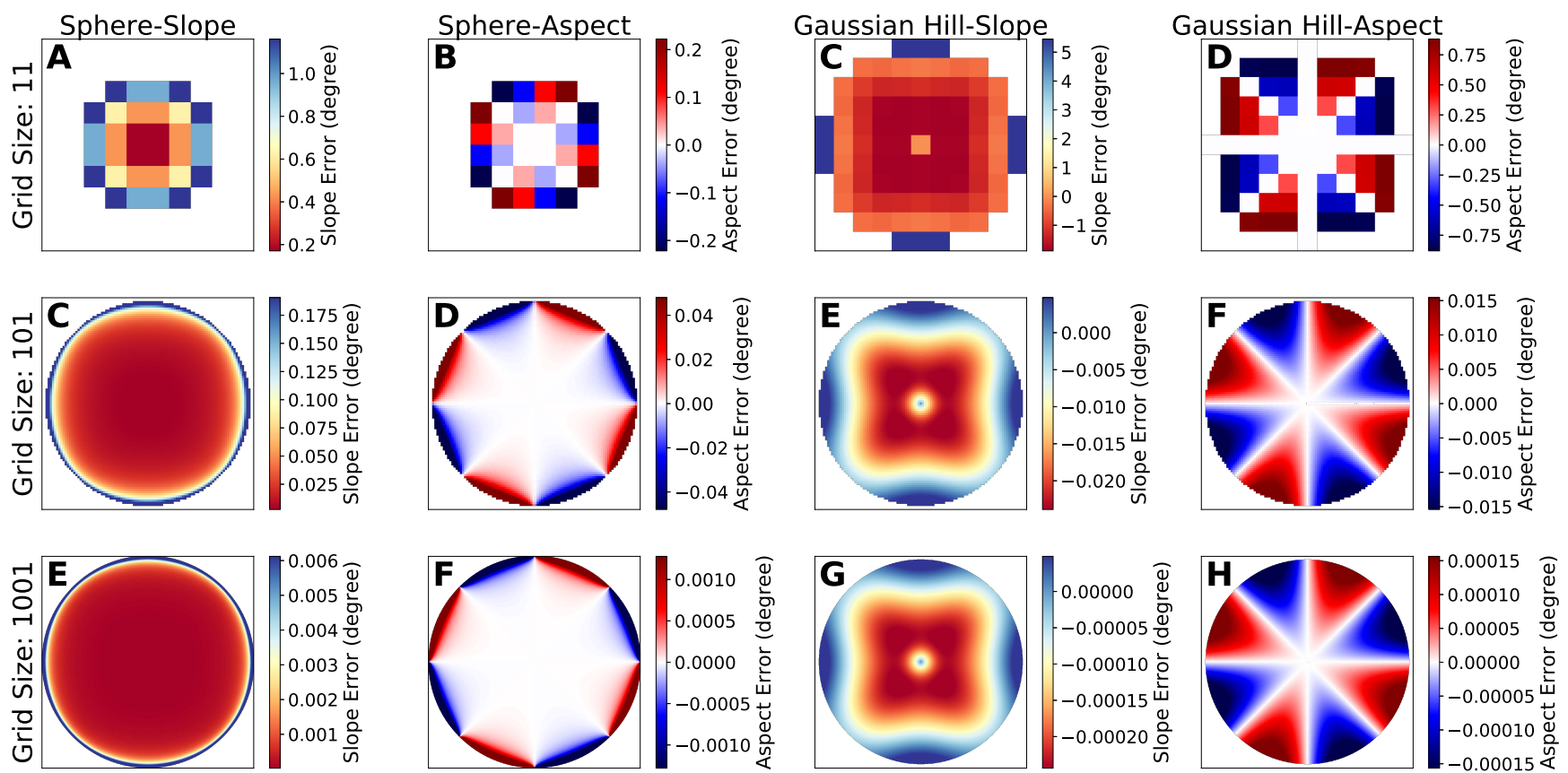

Figure S4. Sphere (left) and Gaussian Hill (right) aspect and slope differences from experimental comparison between calculated and perfect terrain derivatives. Both shapes show clear spatial patterns in slope and aspect biases. While the aspect bias patterns are similar for both shapes, slope differences show distinctly different spatial patterns. Offset magnitudes are generally similar between both shapes, and scale with grid resolution. Colors scaled from 5th to 95th percentiles. 


\section{Sphere}
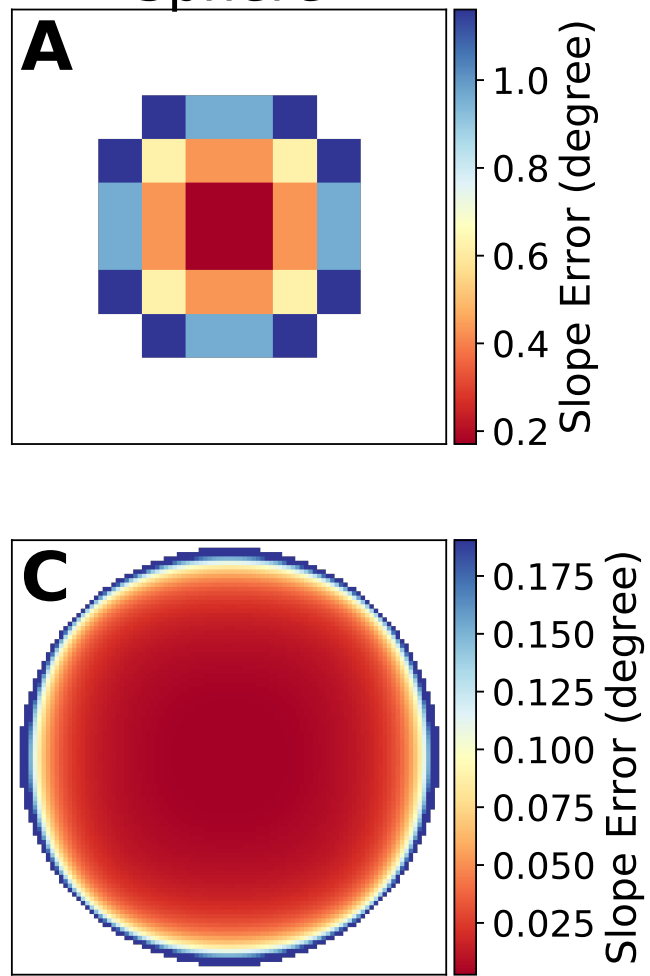

Gaussian Hill

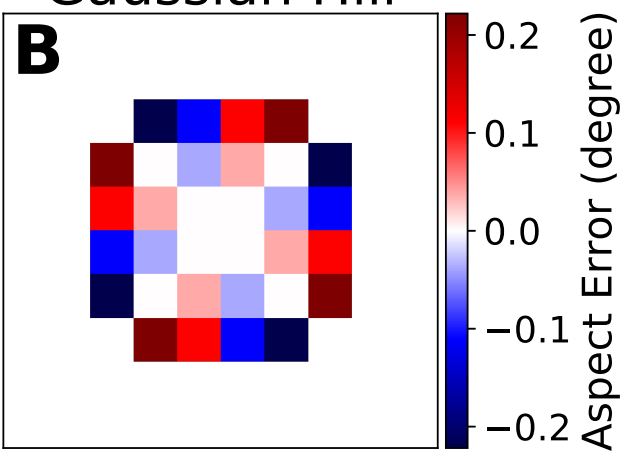

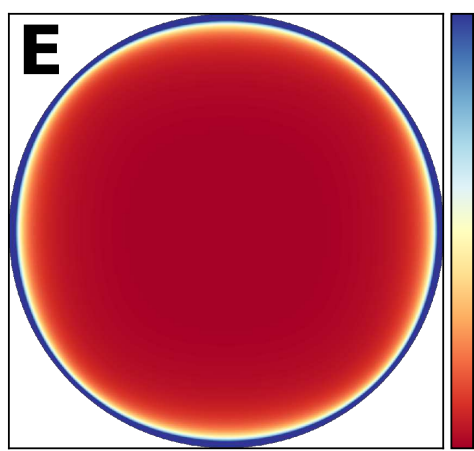
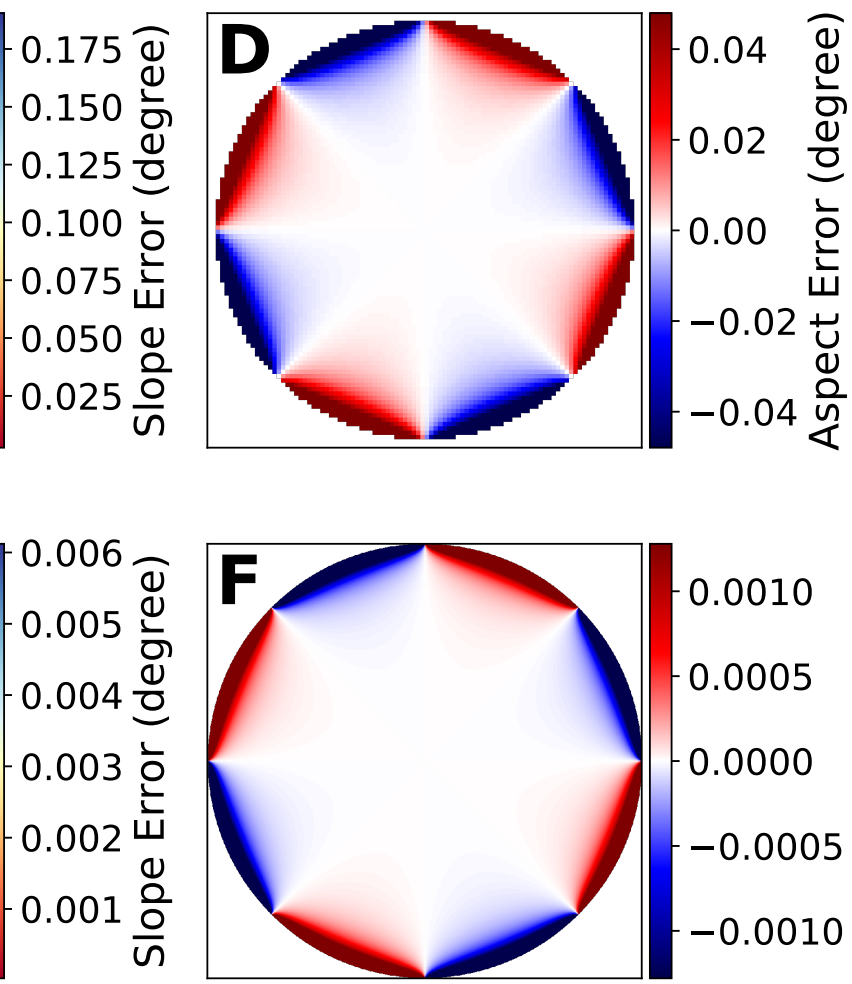

Figure S5. Sphere aspect and slope differences from a mathematically perfect surface, where sphere radius increases with grid size. Spatial and magnitude patterns of offsets are similar between a sphere of fixed radius and one with an increasing radius (cf. Supplemental Figure S4). 

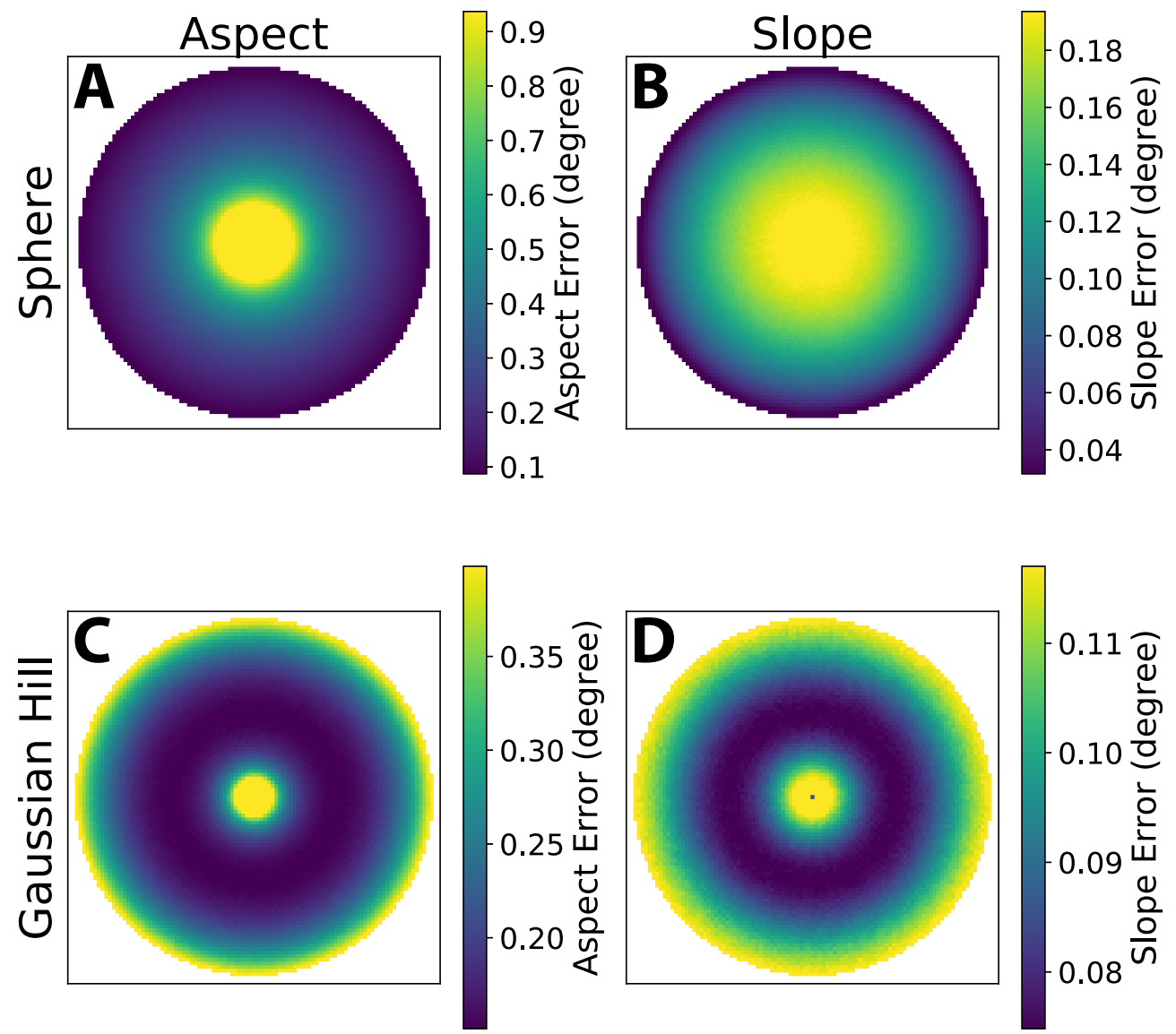

Figure S6. Sphere (left) and Gaussian hill (right) aspect and slope standard deviations for a given noise level, derived from an ensemble of generated DEMs with normally distributed noise (ensemble size, $n=10,000$ ). Noise STD $=1 e^{-4}$. 


\section{Optimal Grid Spacing}

\subsection{Metric Quality Ratios}
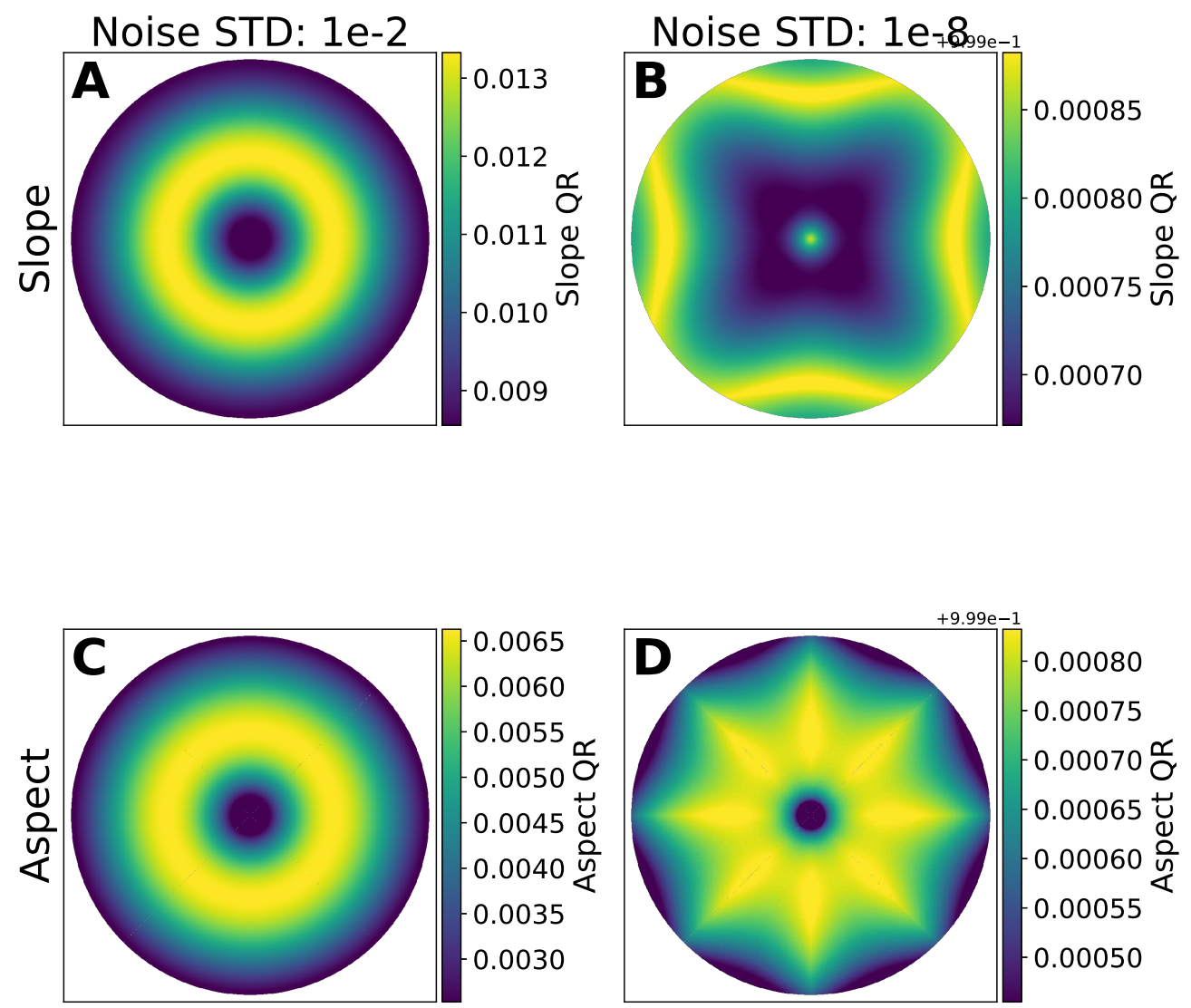

Figure S7. Slope (top) and aspect (bottom) quality ratios for noise standard deviations $1 e^{-2}$ (left) and $1 e^{-8}$ (right). High noise levels lead to error patterns dominated by PEU. Low noise shows the influence of truncation error on the spatial error pattern. 

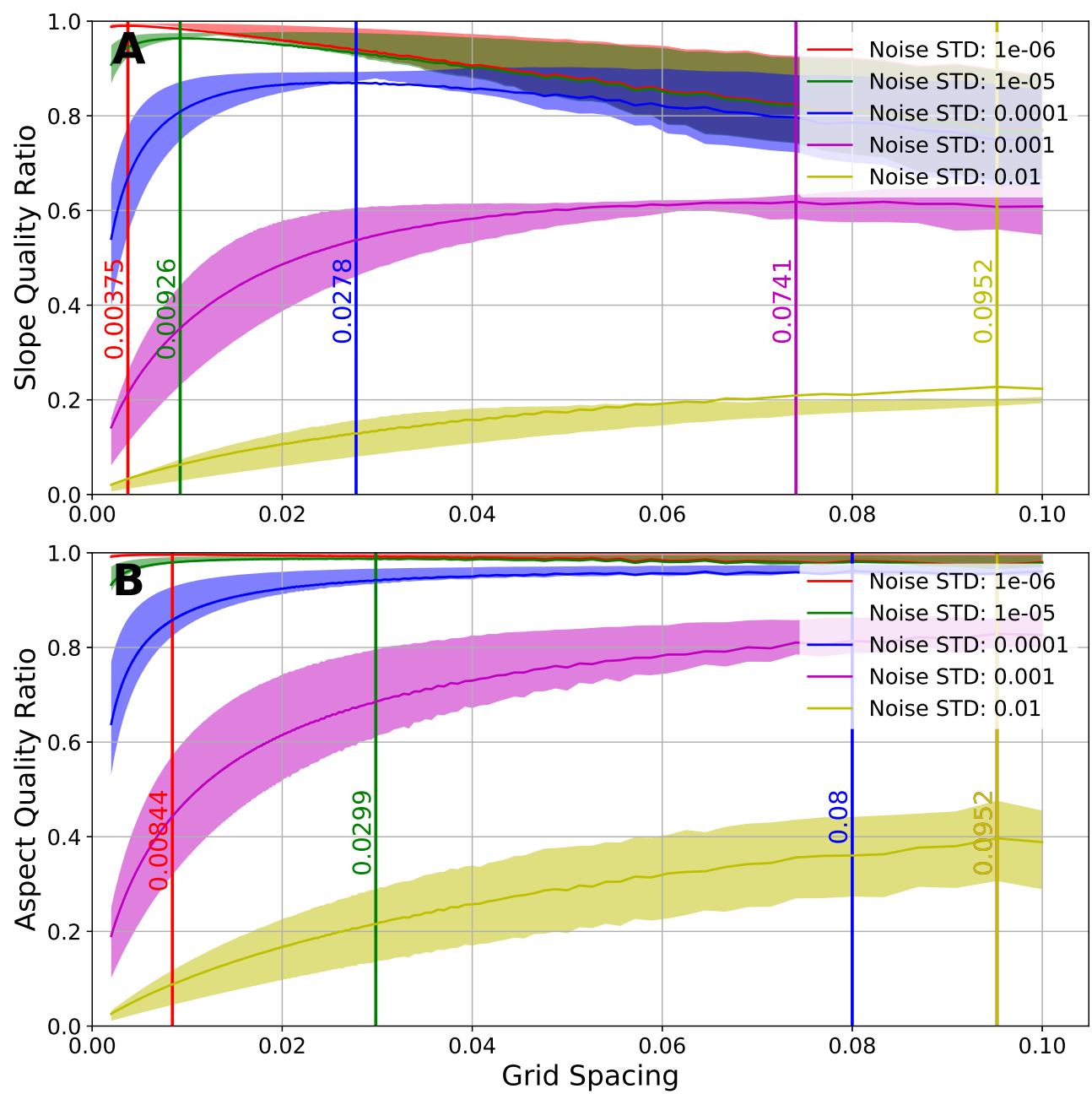

Figure S8. Sphere aspect (top) and slope (bottom) QRs vs grid spacings, for a range of noise levels ( $1 e^{-2}$ to $\left.1 e^{-6}\right) .25$ th-75th percentile QRs shaded for each noise level. Optimal grid resolutions (maximum QR) for each noise level are marked with vertical lines. Slope calculations result in lower optimal grid spacings compared to aspect, because of higher QRs for slope. Higher noise levels lead to higher optimal grid spacings for both slope and aspect calculations. Note that the purple and yellow lines in panel B have the same ideal grid spacing, and thus only the yellow line is visible. 


\subsection{Heterogeneous Noise}
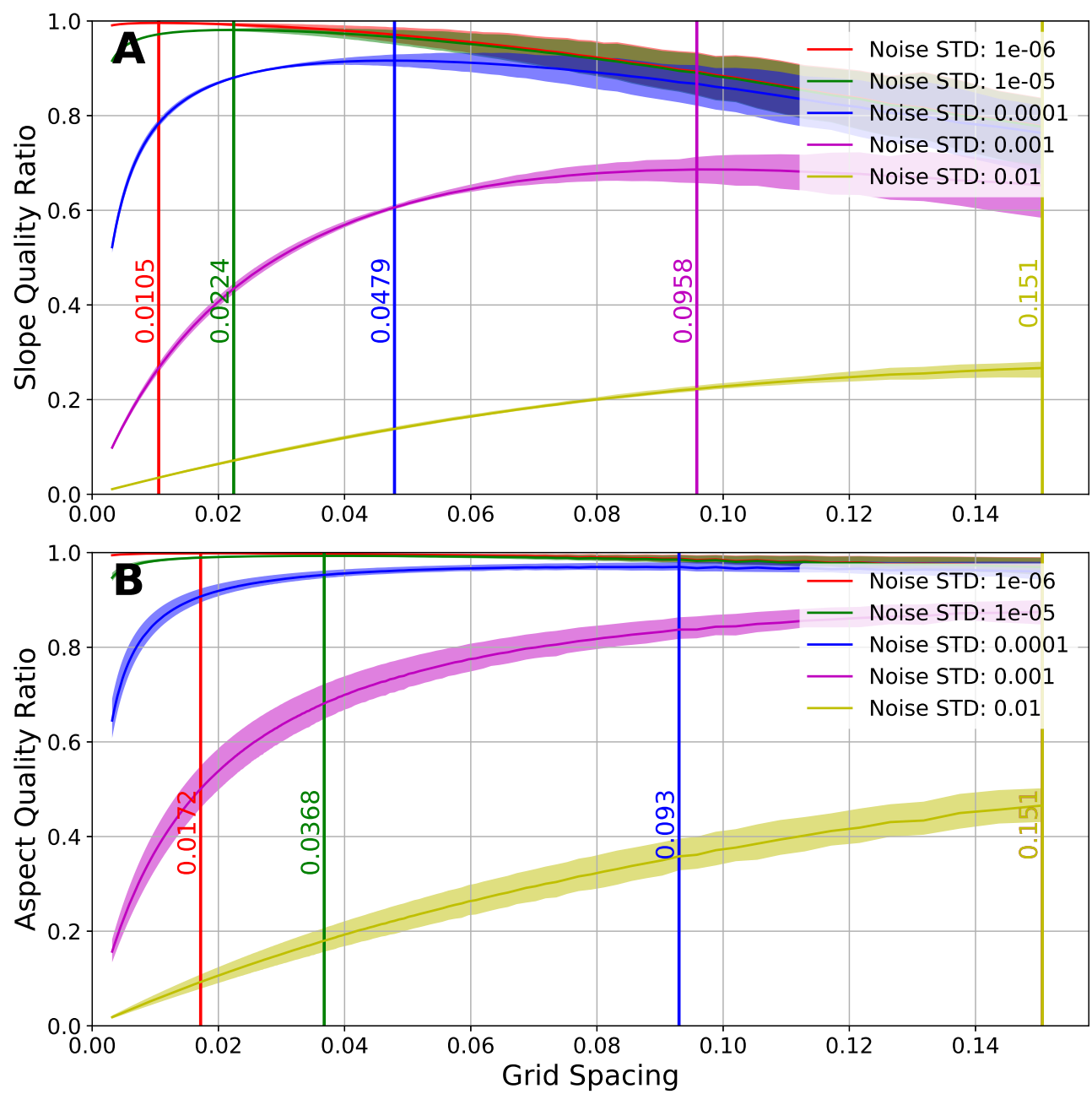

Figure S9. Gaussian hill grid spacing vs QR for varying noise levels biased by slope. 25th-75th percentile QRs shaded. Optimal grid resolution $(\max \mathrm{QR})$ marked with vertical lines. Note that the purple and yellow lines in panel B have the same ideal grid spacing, and thus only the yellow line is visible. 

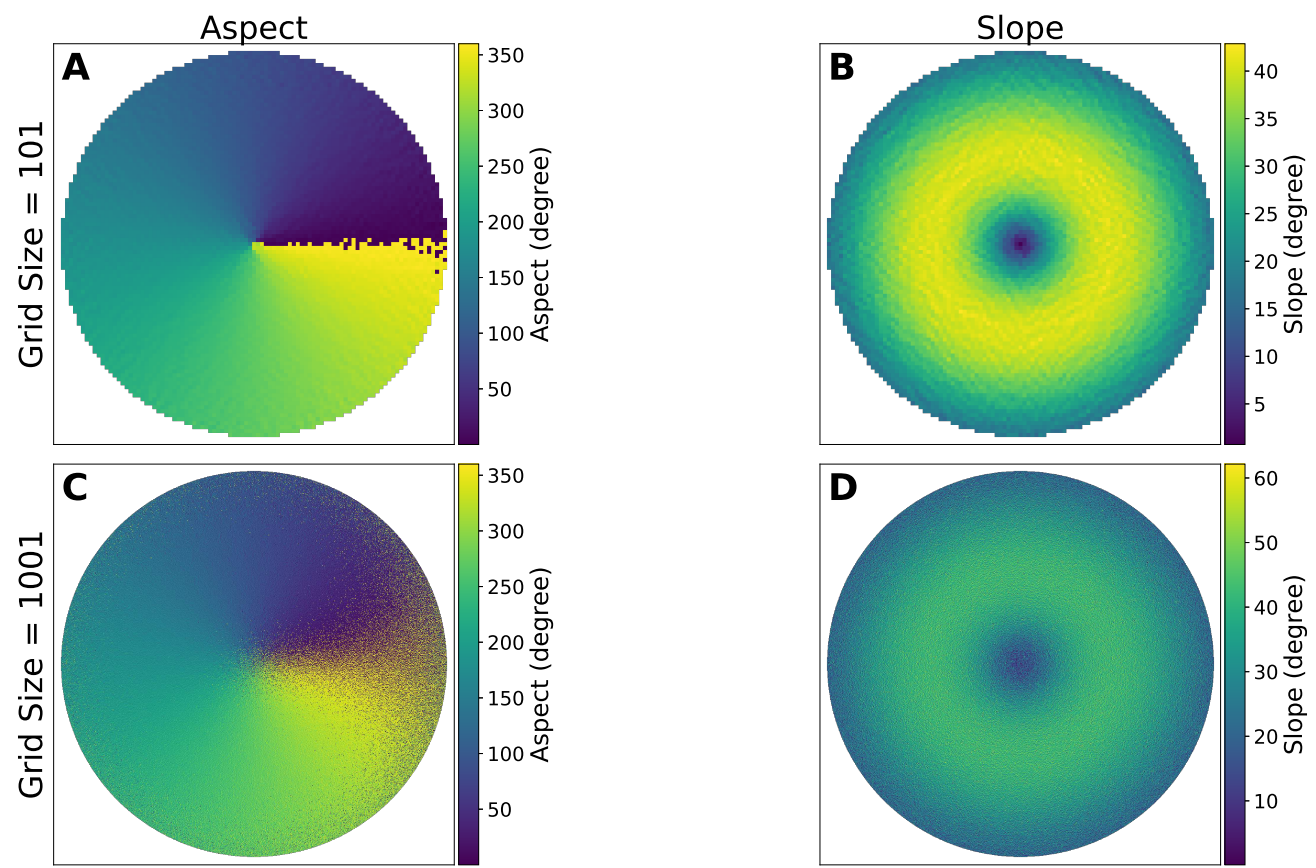

Figure S10. Images showing the Gaussian hill slope and aspect grids with added noise (mean=0, std=1 $\left.e^{-3}\right)$ for $n=101($ top) and $n=1001$ (bottom). 

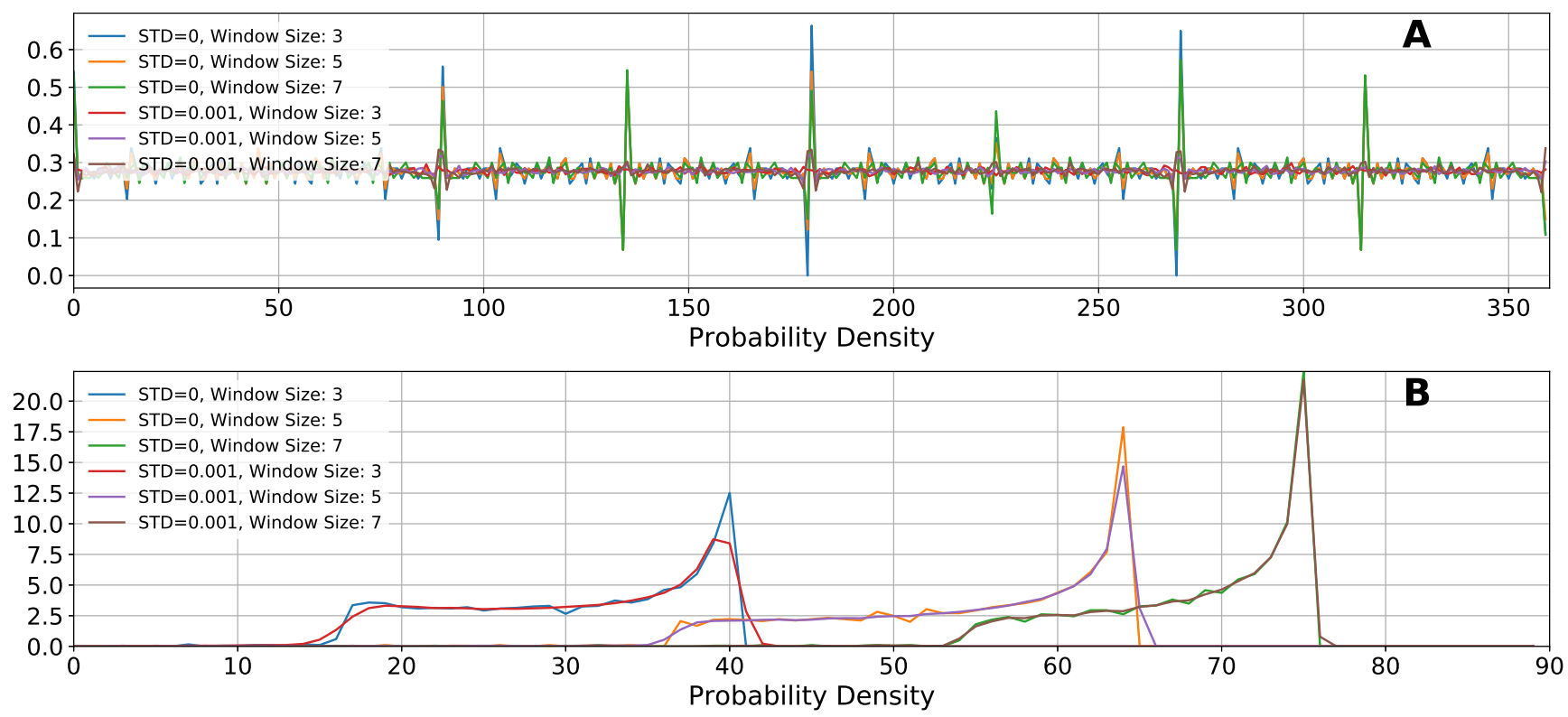

Figure S11. Slope and aspect distributions for a Gaussian hill with variable noise levels and window sizes. 


\section{Case Study: Multi-Resolution Lidar on Santa Cruz Island}

\subsection{Dataset Description}

1 m DEM: Canopy Height Map (CHM)

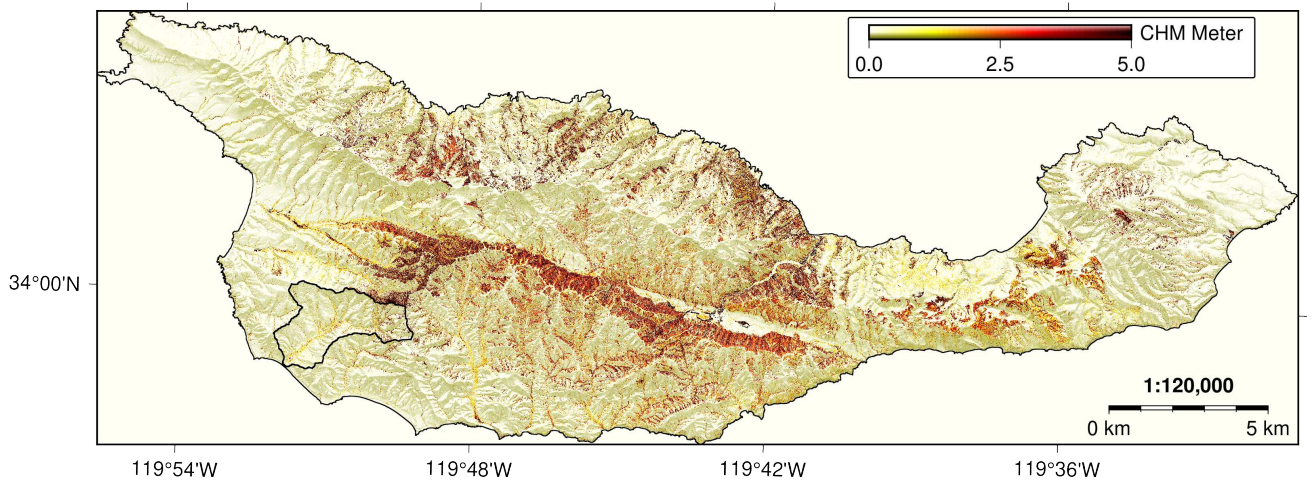

Figure S12. Island-wide canopy-height model, derived from a one meter DEM (cf. Figure 10). Vegetation heights are generally low, excepting some central regions of SCI. 


\subsection{Deriving Elevations and Elevation Uncertainties}

\subsubsection{Alternative Interpolation Schemes}

Table S1. Listing of interpolation schemes used to create DEMs for SCI. For a more in-depth discussion of each method, we refer to our github page: https://github.com/UP-RS-ESP/TopoMetricUncertainty.

\begin{tabular}{|l|l|l|}
\hline Scheme Name & Software/Algorithm Basis & \multirow{2}{*}{ Figure } \\
\hline Triangular Irregular Network & blast2dem, LAStools, LAStools (2017) & \\
\hline Triangular Irregular Network, Delauney Method & triangulation, GMT, Watson (1982) & Figure S13 \\
\hline Local Mean & blockmean, GMT, Wessel and Luis (2017) & Figure S14 \\
\hline Local Median & blockmedian, GMT, Wessel and Luis (2017) & Figure S15 \\
\hline Inverse Distance Weights, power=1, radius=grid size $\times \frac{\sqrt{2}}{2}$ & gdal_grid, GDAL (gdal.org), Shepard (1968) & Figure S16 \\
\hline Inverse Distance Weights, power=1, radius=grid size $\times \sqrt{2}$ & gda__grid, GDAL (gdal.org), Shepard (1968) & Figure S17 \\
\hline Inverse Distance Weights, power=1, radius=grid size $\times 2 \sqrt{2}$ & gdal_grid, GDAL (gdal.org), Shepard (1968) & Figure S18 \\
\hline Inverse Distance Weights, power=2, radius=grid size $\times \frac{\sqrt{2}}{2}$ & gdal_grid, GDAL (gdal.org), Shepard (1968) & Figure S19 \\
\hline Inverse Distance Weights, power=3, radius=grid size $\times \frac{\sqrt{2}}{2}$ & gdal_grid, GDAL (gdal.org), Shepard (1968) & Figure S20 \\
\hline Inverse Distance Weights, power=2, radius=grid size $\times \frac{\sqrt{2}}{2}$ & Points2Grid, pdal (pdal.io), Shepard (1968) & Figure S21 \\
\hline
\end{tabular}



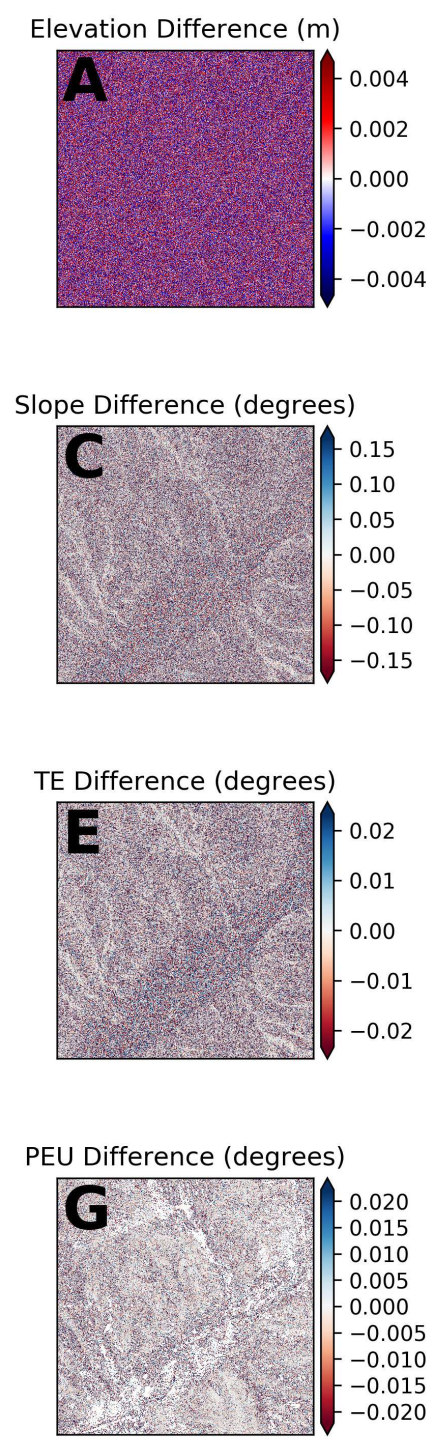

25th, 50th, 75th Percentile: $-0.00253,0,0.00252$

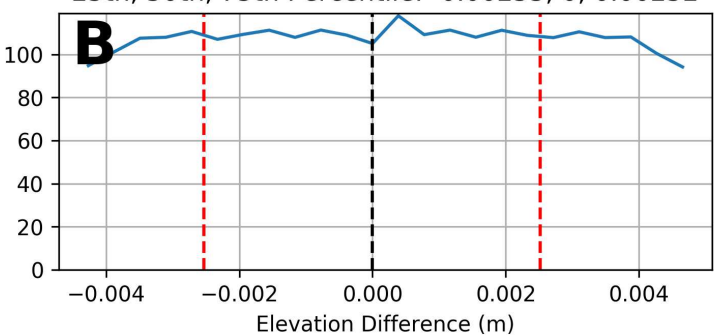

25th, 50th, 75th Percentile: $-0.0649,-0.00035,0.0643$

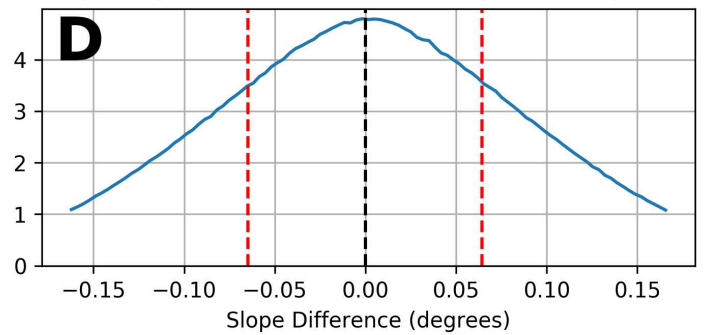

25th, 50th, 75th Percentile: $-0.00839,6.76 e-05,0.00853$

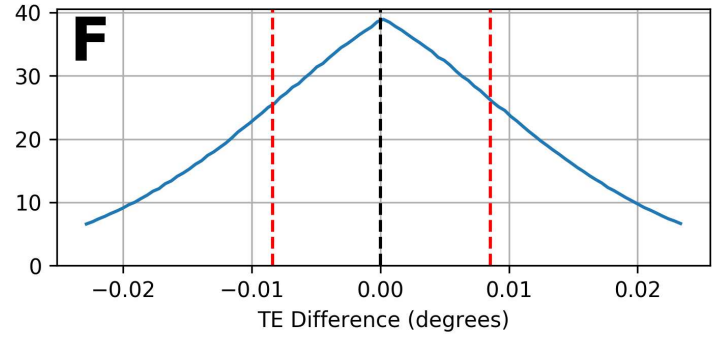

25th, 50th, 75th Percentile: $-0.00696,2.52 e-05,0.00701$

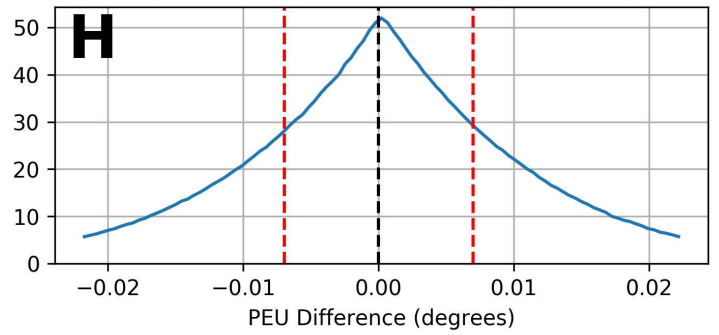

Figure S13. Pozo catchment one meter DEM difference between the LAStools TIN method LAStools (2017) and a one meter DEM generated using a Delauney triangulation interpolation scheme (triangulation function, Watson (1982)). Color bar scaled from 5th to 95th percentiles. Right column shows the density histogram of the differences for the whole Pozo catchment. Red lines represent the 25th and 75th percentiles, black line on zero. Histograms scaled between 5th and 95th percentiles. We refer to our github page for more detailed processing steps: https:/github.com/UP-RS-ESP/TopoMetricUncertainty. 

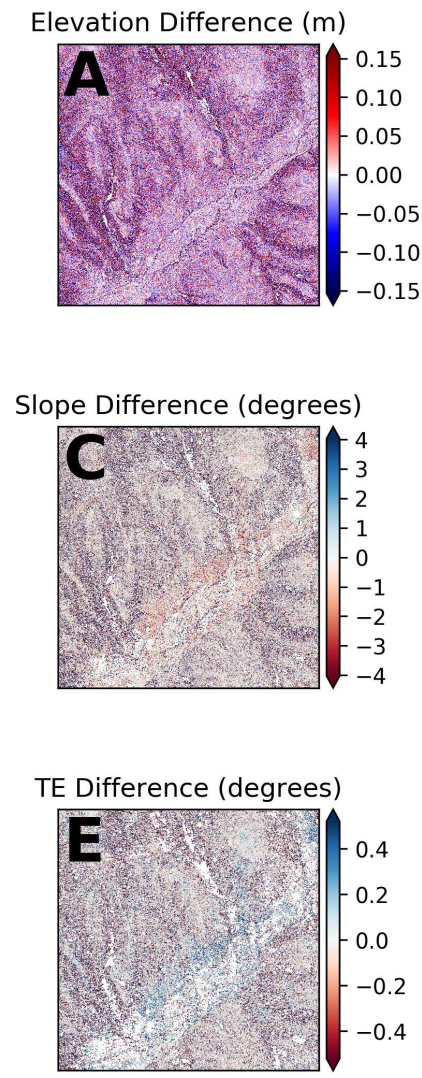

PEU Difference (degrees)

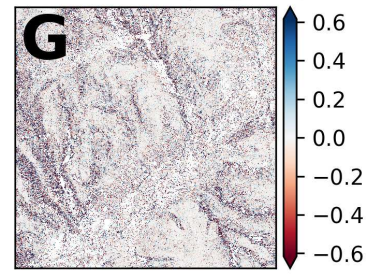

25th, 50th, 75th Percentile: $-0.0433,0,0.04$

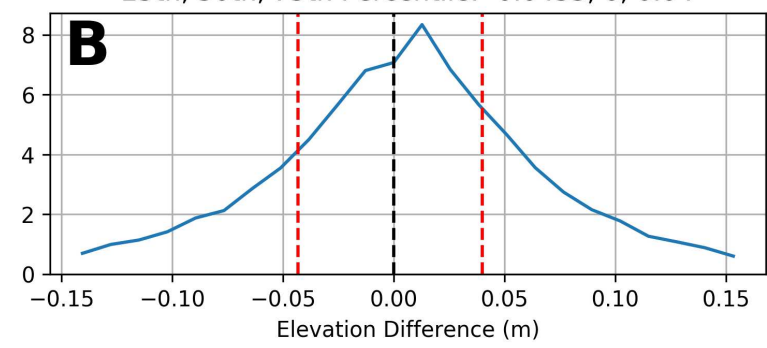

25th, 50th, 75th Percentile: $-1.41,-0.0571,1.3$

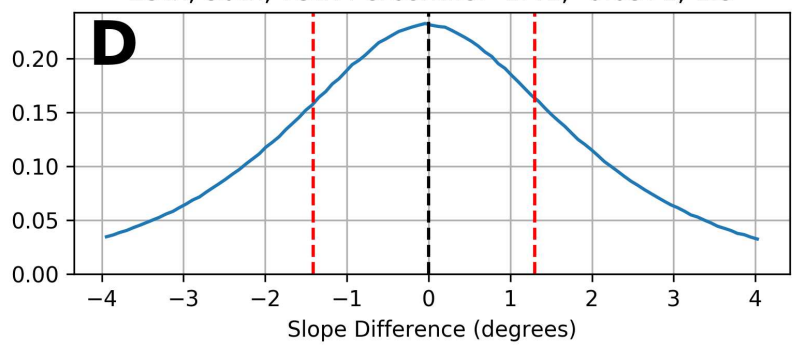

25th, 50th, 75th Percentile: $-0.167,0.0081,0.183$

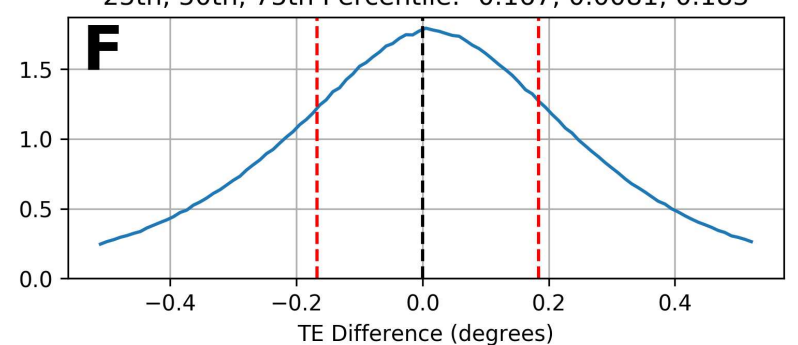

25th, 50th, 75th Percentile: $-0.131,0.00315,0.143$

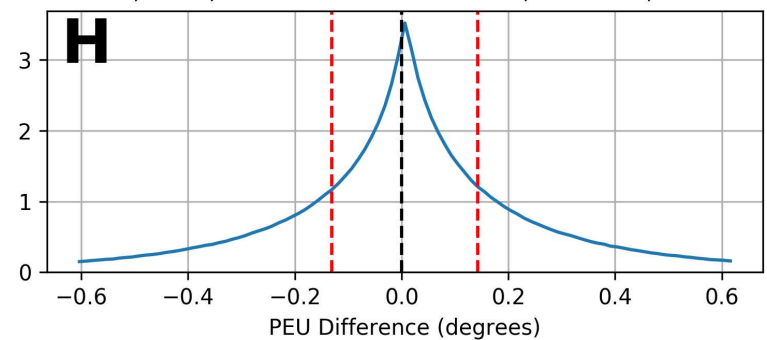

Figure S14. Pozo catchment one meter DEM difference between the LAStools TIN method LAStools (2017) and a one meter DEM generated using the mean of all points within a grid cell (blockmean function, Wessel and Luis (2017)). Color bar scaled from 5th to 95th percentiles. Right column shows the density histogram of the differences for the whole Pozo catchment. Red lines represent the 25th and 75th percentiles, black line on zero. Histograms scaled between 5th and 95th percentiles. We refer to our github page for more detailed processing steps: https://github.com/UP-RS-ESP/TopoMetricUncertainty. 

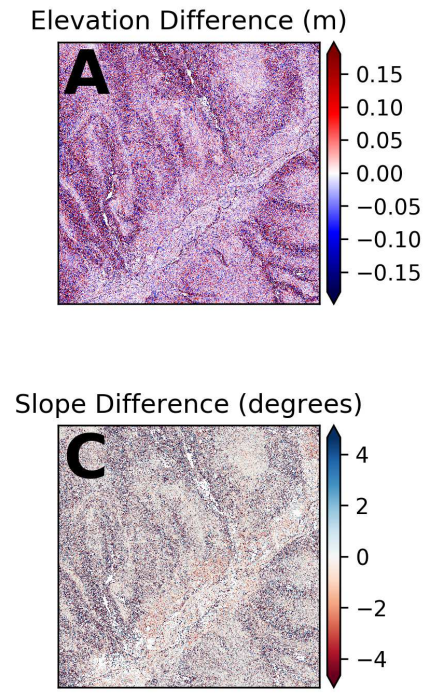

TE Difference (degrees)

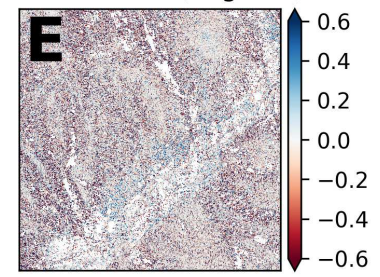

PEU Difference (degrees)

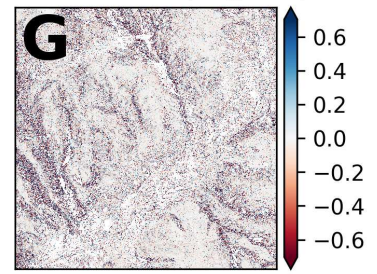

25th, 50th, 75th Percentile: $-0.05,0,0.04$

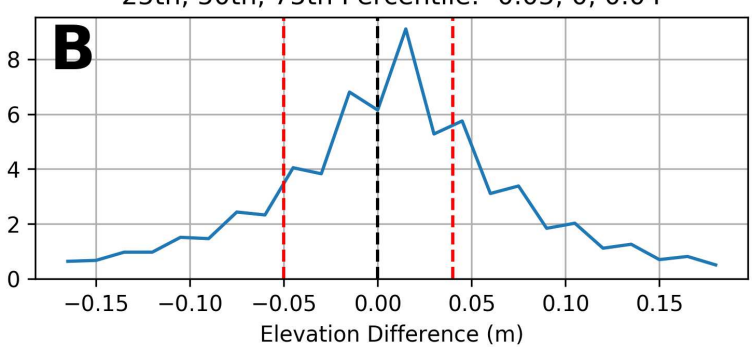

25th, 50th, 75th Percentile: -1.52, 2.69e-05, 1.53

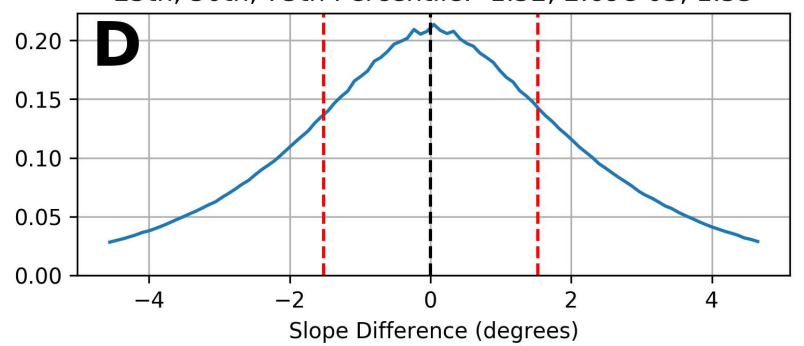

25th, 50th, 75th Percentile: $-0.202,-0.0048,0.193$

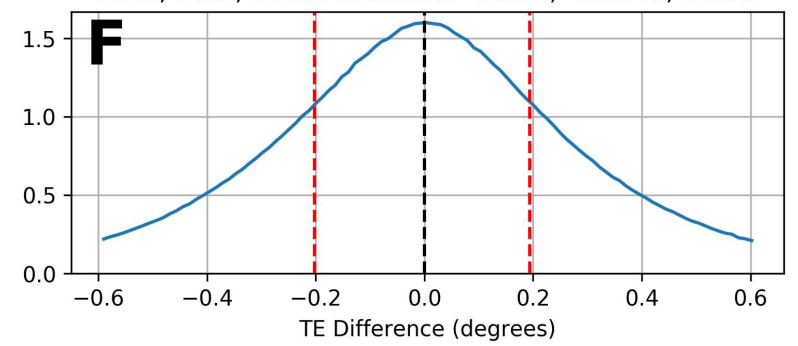

25th, 50th, 75th Percentile: $-0.155,1.92 \mathrm{e}-05,0.152$

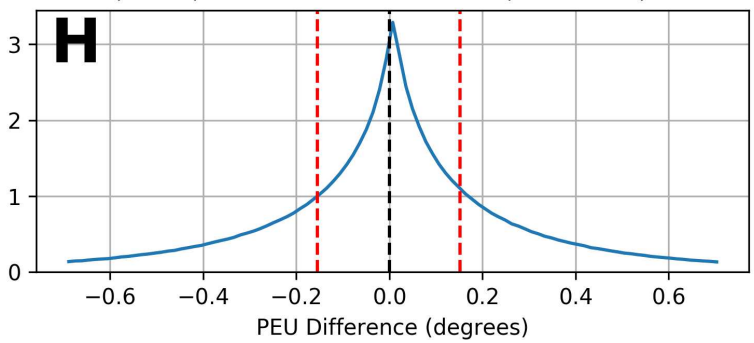

Figure S15. Pozo catchment one meter DEM difference between the LAStools TIN method LAStools (2017) and a one meter DEM generated using the median of all points within a grid cell (blockmedian function, Wessel and Luis (2017)). Color bar scaled from 5th to 95th percentiles. Right column shows the density histogram of the differences for the whole Pozo catchment. Red lines represent the 25th and 75th percentiles, black line on zero. Histograms scaled between 5th and 95th percentiles. We refer to our github page for more detailed processing steps: https:/github.com/UP-RS-ESP/TopoMetricUncertainty. 

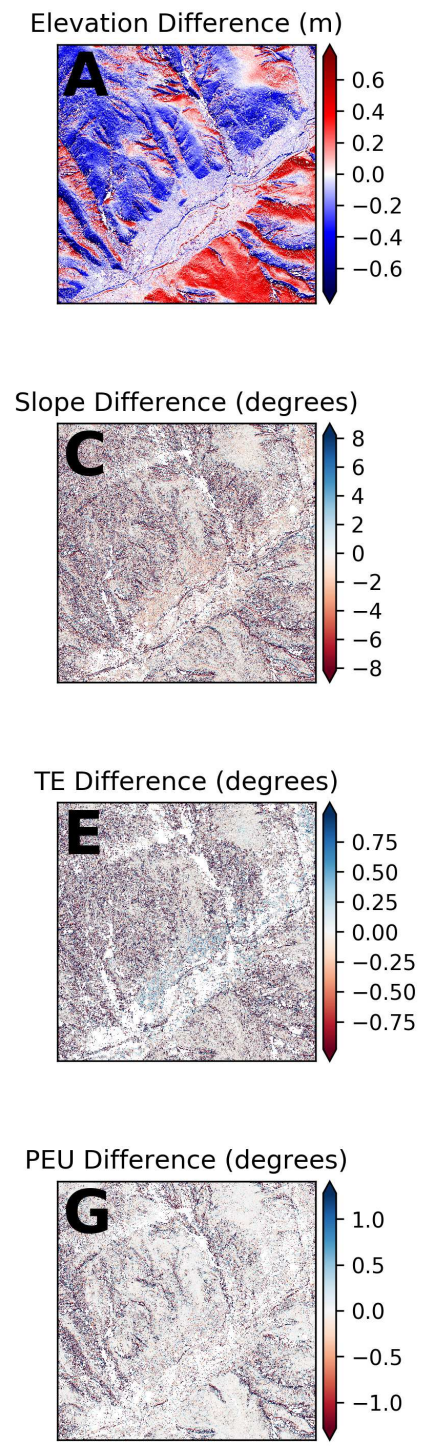

25th, 50th, 75th Percentile: $-0.336,-0.071,0.21$

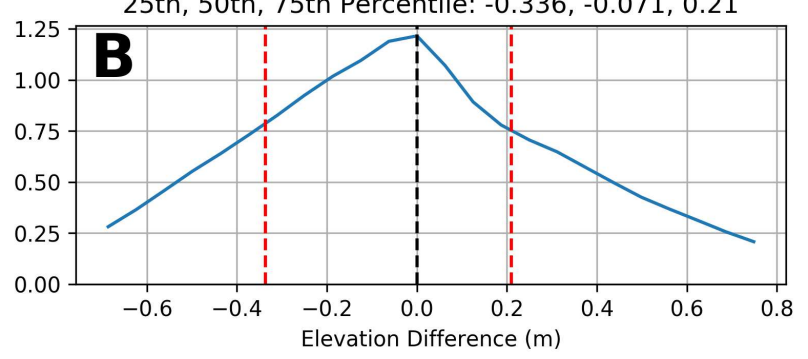

25th, 50th, 75th Percentile: $-2.66,-0.135,2.38$

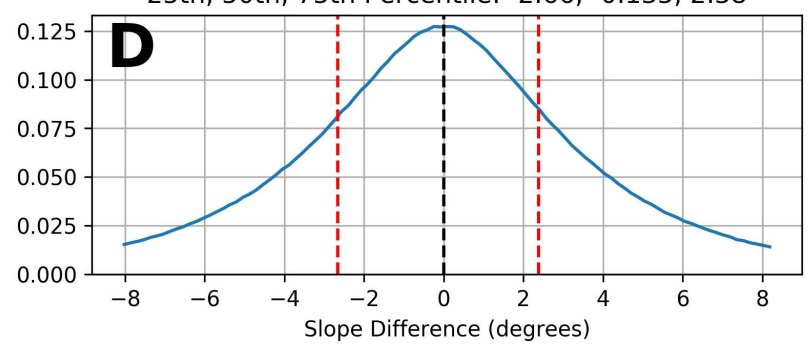

25th, 50th, 75th Percentile: $-0.289,0.019,0.331$

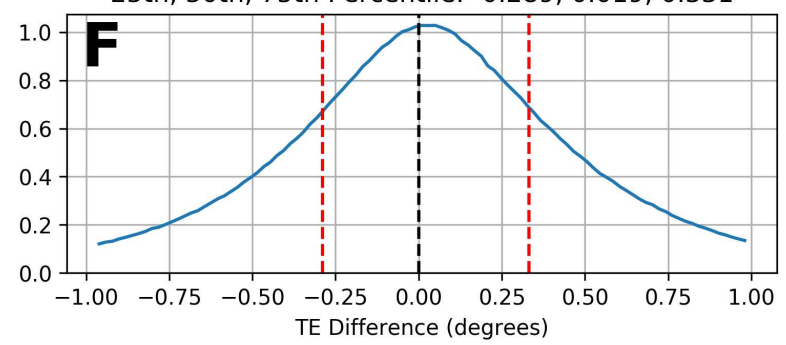

25th, 50th, 75th Percentile: $-0.239,0.00851,0.28$

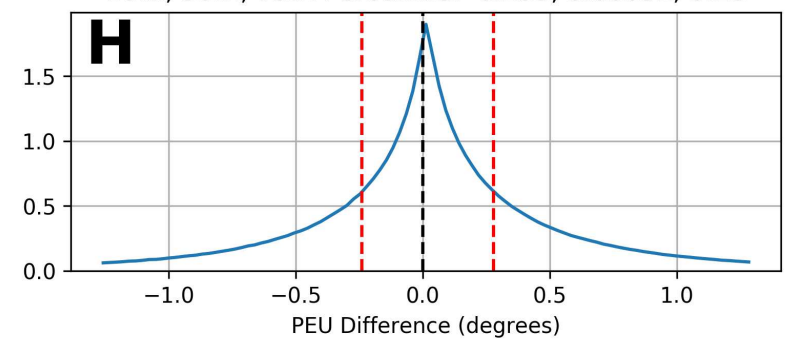

Figure S16. Pozo catchment one meter DEM difference between the LAStools TIN method LAStools (2017) and a one meter DEM generated using an inverse distance weighting (power=1, radius=grid size $\times \frac{\sqrt{2}}{2}$ ) (gdal_grid function, gdal.org, Shepard (1968)). Color bar scaled from 5th to 95th percentiles. Right column shows the density histogram of the differences for the whole Pozo catchment. Red lines represent the 25th and 75th percentiles, black line on zero. Histograms scaled between 5th and 95th percentiles. We refer to our github page for more detailed processing steps: https://github.com/UP-RS-ESP/TopoMetricUncertainty. 

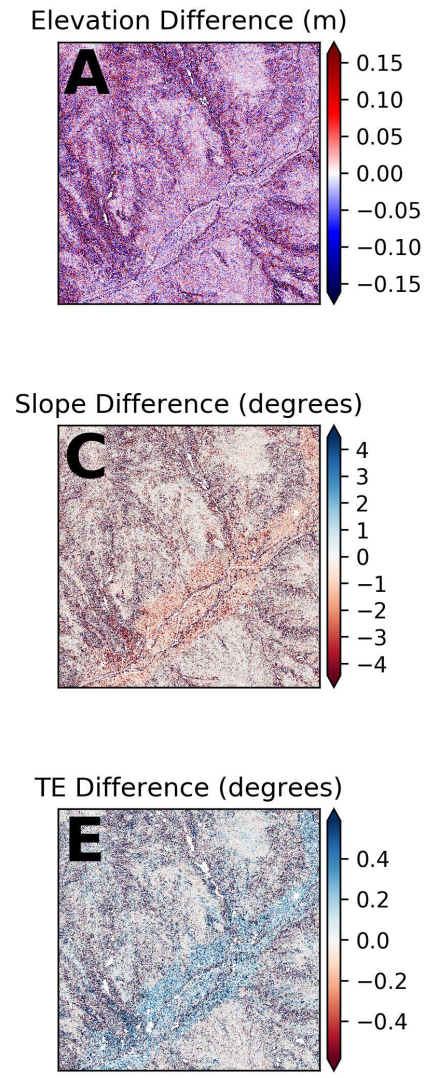

PEU Difference (degrees)

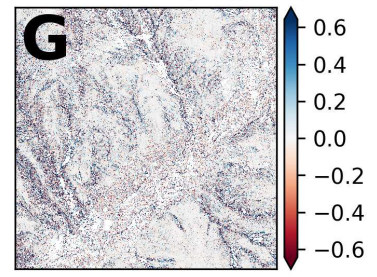

25th, 50th, 75th Percentile: $-0.0434,-0.00237,0.034$

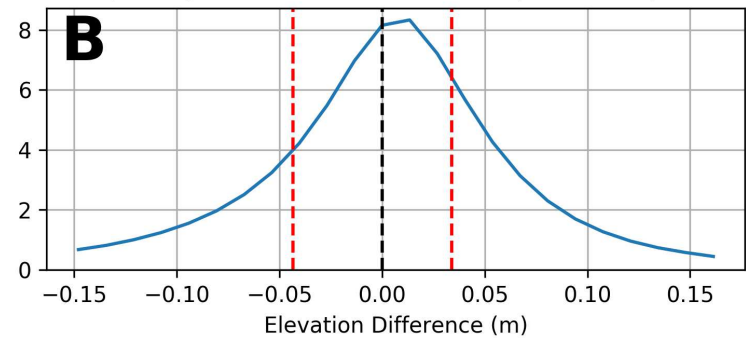

25th, 50th, 75th Percentile: $-1.57,-0.272,0.95$

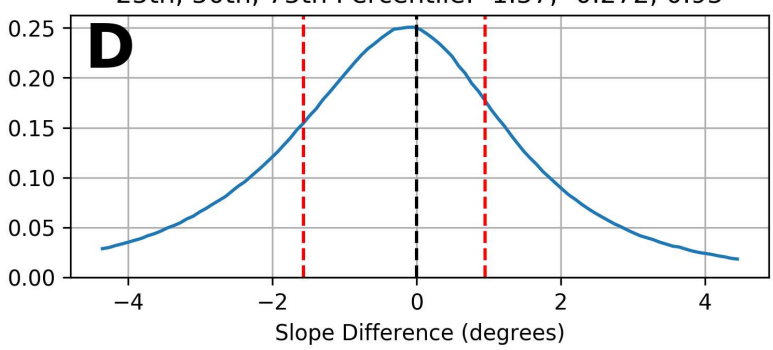

25th, 50th, 75th Percentile: $-0.114,0.0459,0.214$

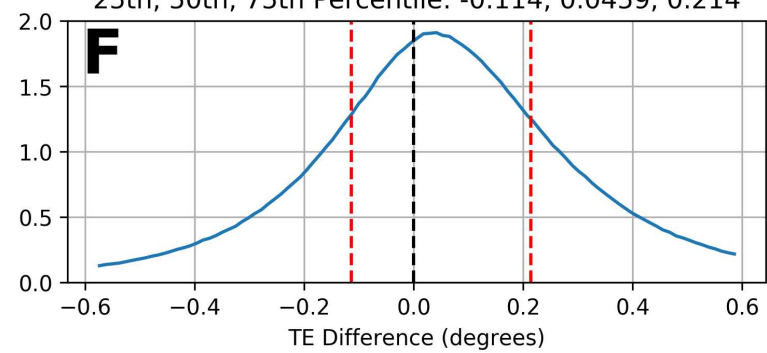

25th, 50th, 75th Percentile: $-0.0846,0.0173,0.159$

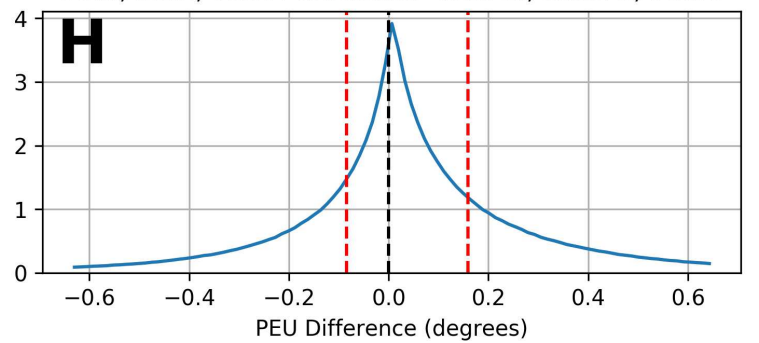

Figure S17. Pozo catchment one meter DEM difference between the LAStools TIN method LAStools (2017) and a one meter DEM generated using an inverse distance weighting (power=1, radius=grid size $\times \sqrt{2}$ ) (gdal_grid function, gdal.org, Shepard (1968)). Color bar scaled from 5th to 95th percentiles. Right column shows the density histogram of the differences for the whole Pozo catchment. Red lines represent the 25 th and 75th percentiles, black line on zero. Histograms scaled between 5th and 95th percentiles. We refer to our github page for more detailed processing steps: https://github.com/UP-RS-ESP/TopoMetricUncertainty. 

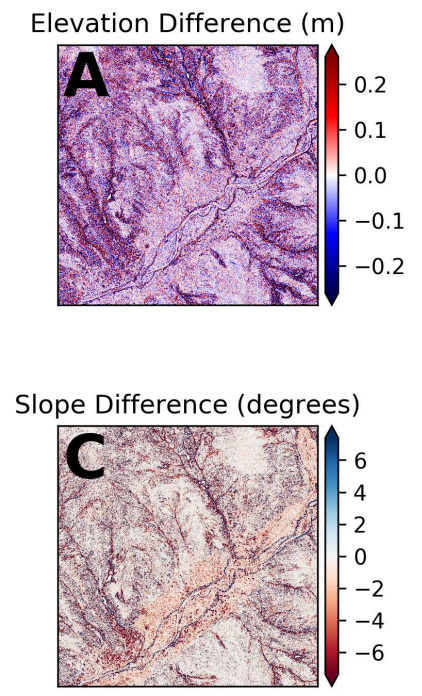

TE Difference (degrees)

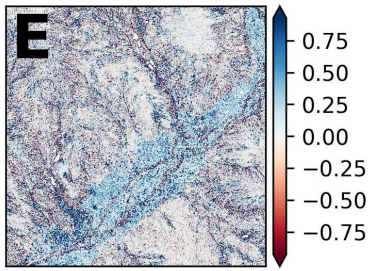

PEU Difference (degrees)

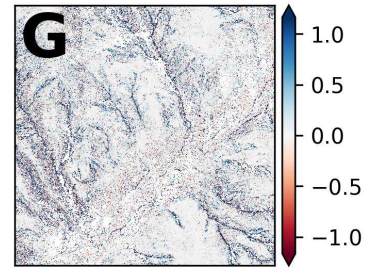

25th, 50th, 75th Percentile: $-0.0691,-0.00562,0.0506$

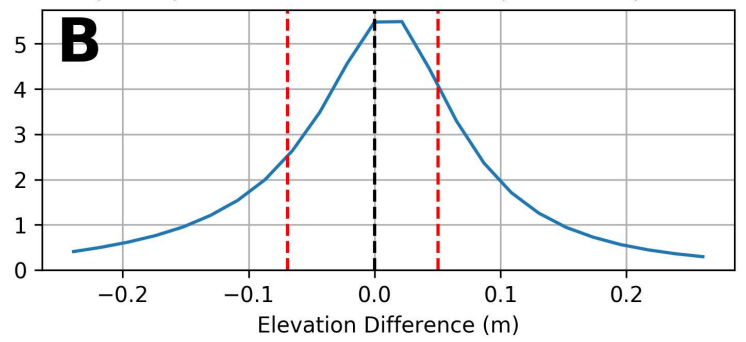

25th, 50th, 75th Percentile: $-2.51,-0.531,1.21$

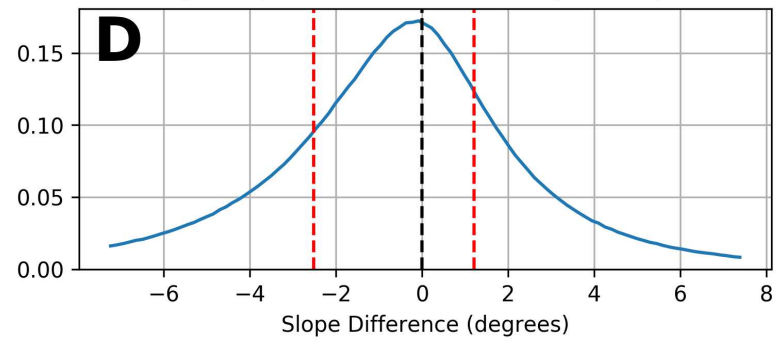

25th, 50th, 75th Percentile: $-0.144,0.0862,0.343$

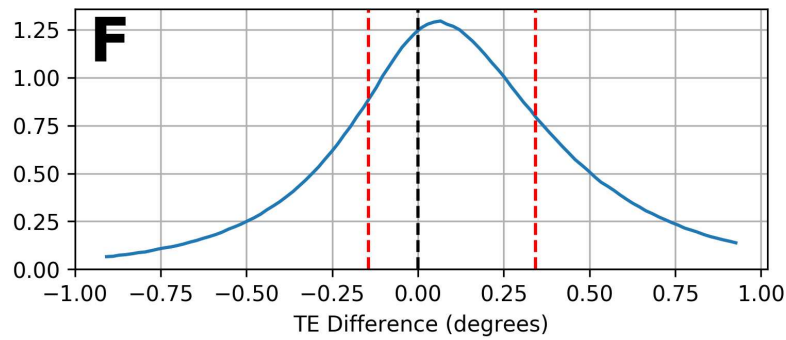

25th, 50th, 75th Percentile: $-0.0972,0.0391,0.276$

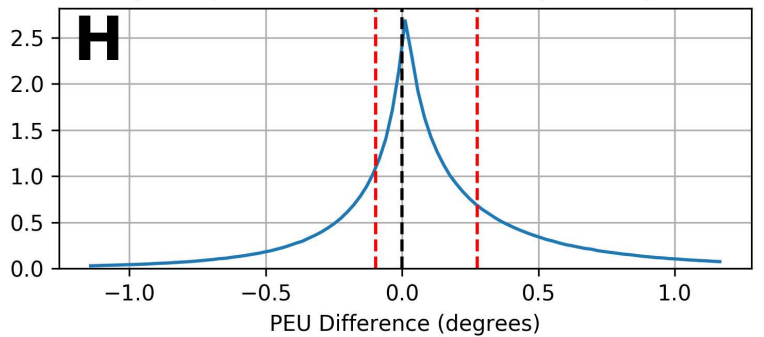

Figure S18. Pozo catchment one meter DEM difference between the LAStools TIN method LAStools (2017) and a one meter DEM generated using an inverse distance weighting (power $=1$, radius = grid size $\times 2 \sqrt{2}$ ) (gdal_grid function, gdal.org, Shepard (1968)). Color bar scaled from 5th to 95th percentiles. Right column shows the density histogram of the differences for the whole Pozo catchment. Red lines represent the 25 th and 75 th percentiles, black line on zero. Histograms scaled between 5th and 95th percentiles. We refer to our github page for more detailed processing steps: https://github.com/UP-RS-ESP/TopoMetricUncertainty. 

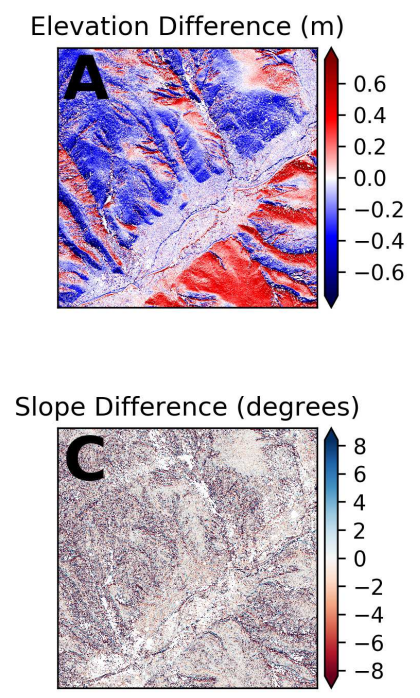

TE Difference (degrees)

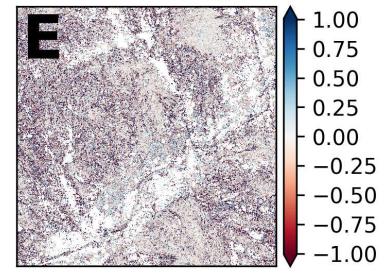

PEU Difference (degrees)

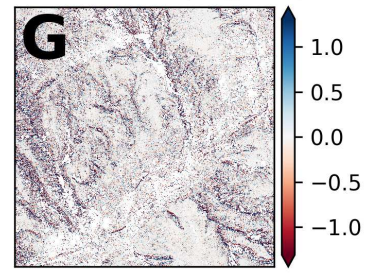

25th, 50th, 75th Percentile: $-0.337,-0.0698,0.212$

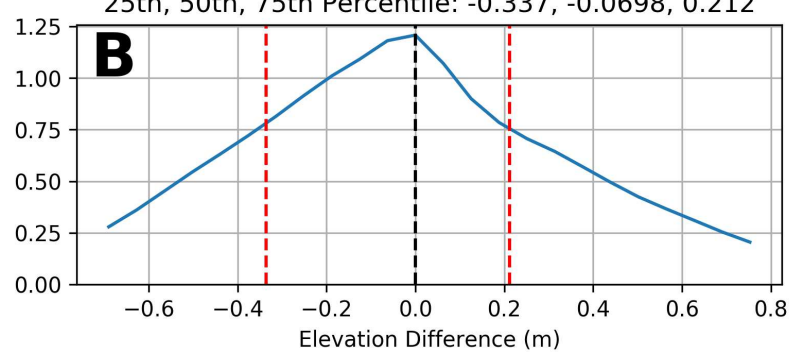

25th, 50th, 75th Percentile: $-2.72,-0.0775,2.56$

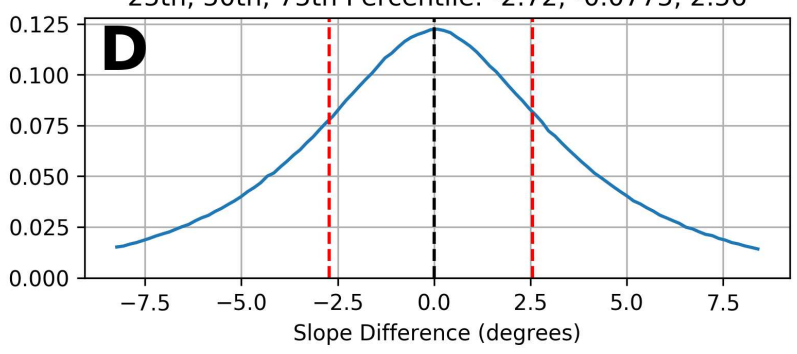

25th, 50th, 75th Percentile: $-0.316,0.00767,0.334$

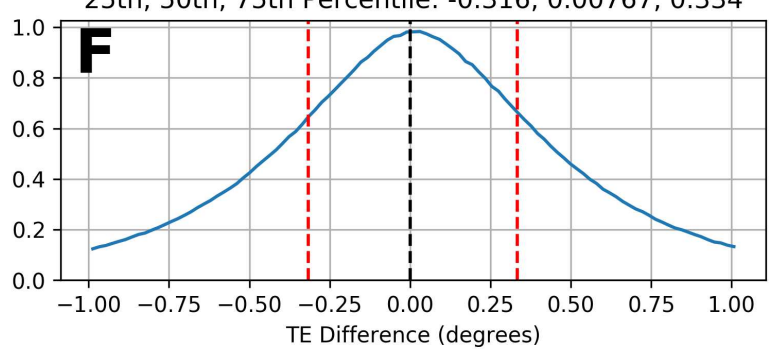

25th, 50th, 75th Percentile: $-0.258,0.00519,0.284$

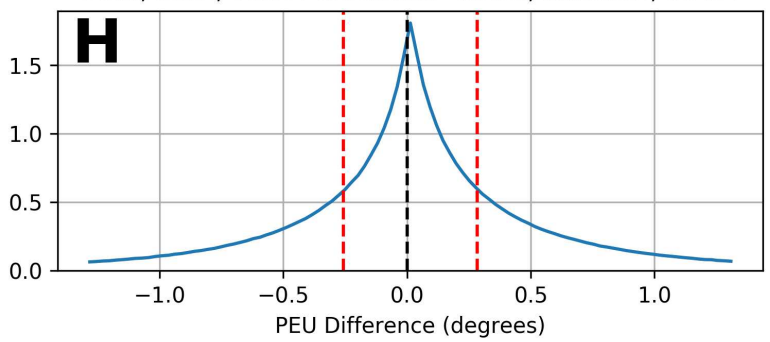

Figure S19. Pozo catchment one meter DEM difference between the LAStools TIN method LAStools (2017) and a one meter DEM generated using an inverse distance weighting (power=2, radius=grid size $\times \frac{\sqrt{2}}{2}$ ) (gdal_grid function, gdal.org, Shepard (1968)). Color bar scaled from 5th to 95th percentiles. Right column shows the density histogram of the differences for the whole Pozo catchment. Red lines represent the 25th and 75th percentiles, black line on zero. Histograms scaled between 5th and 95th percentiles. We refer to our github page for more detailed processing steps: https://github.com/UP-RS-ESP/TopoMetricUncertainty. 

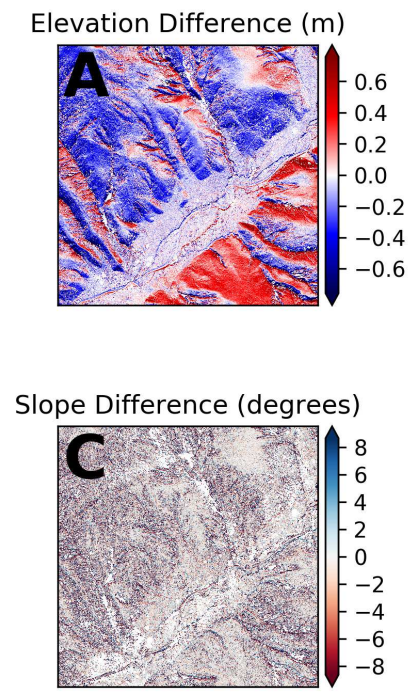

TE Difference (degrees)

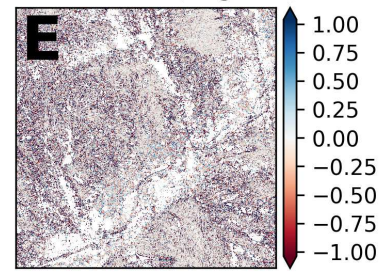

PEU Difference (degrees)

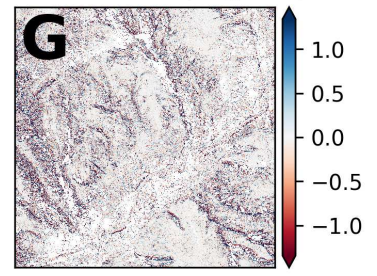

25th, 50th, 75th Percentile: $-0.337,-0.0689,0.213$

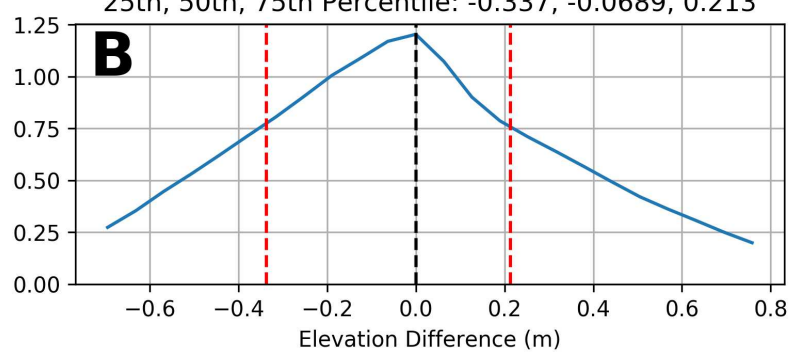

25th, 50th, 75th Percentile: $-2.79,-0.0263,2.75$

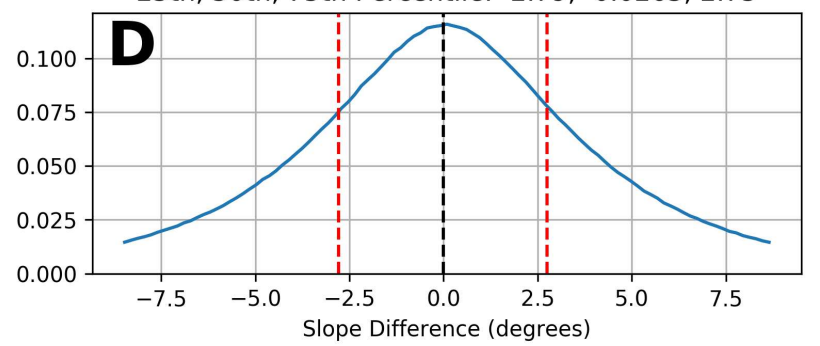

25th, 50th, 75th Percentile: $-0.345,-0.00336,0.34$

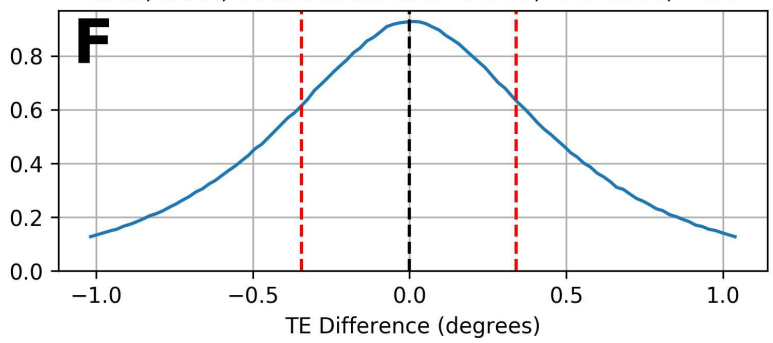

25th, 50th, 75th Percentile: $-0.278,0.00233,0.291$

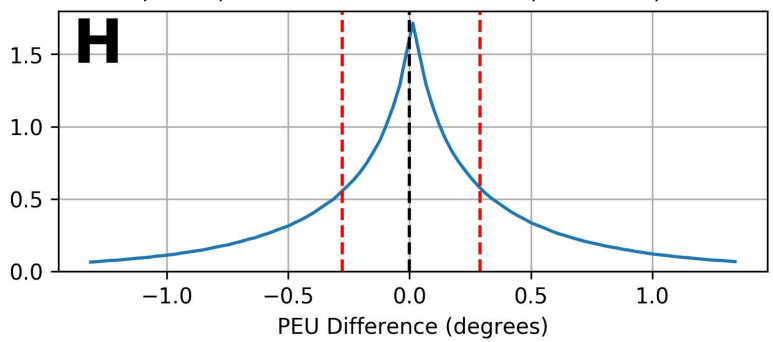

Figure S20. Pozo catchment one meter DEM difference between the LAStools TIN method LAStools (2017) and a one meter DEM generated using an inverse distance weighting (power=3, radius=grid size $\times \frac{\sqrt{2}}{2}$ ) (gdal_grid function, gdal.org, Shepard (1968)). Color bar scaled from 5th to 95th percentiles. Right column shows the density histogram of the differences for the whole Pozo catchment. Red lines represent the 25th and 75th percentiles, black line on zero. Histograms scaled between 5th and 95th percentiles. We refer to our github page for more detailed processing steps: https://github.com/UP-RS-ESP/TopoMetricUncertainty. 

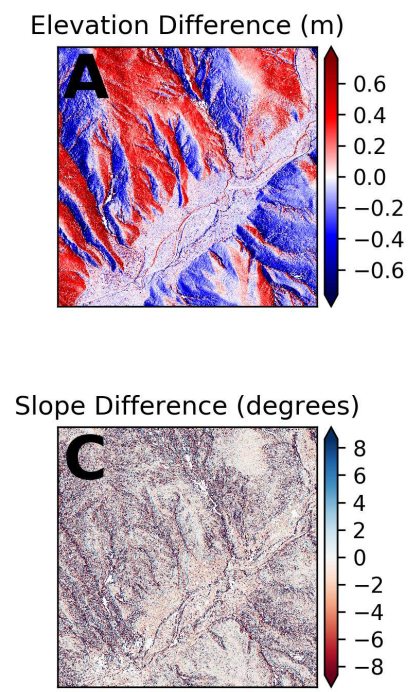

TE Difference (degrees)

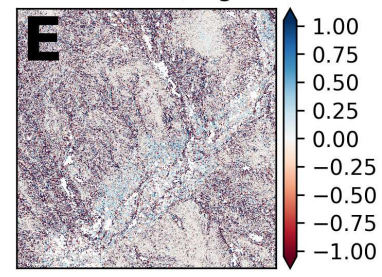

PEU Difference (degrees)

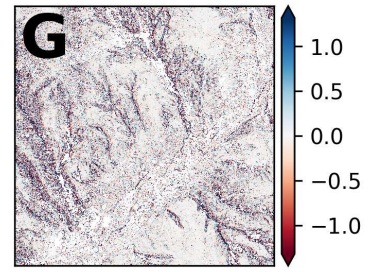

25th, 50th, 75th Percentile: $-0.337,-0.0457,0.24$

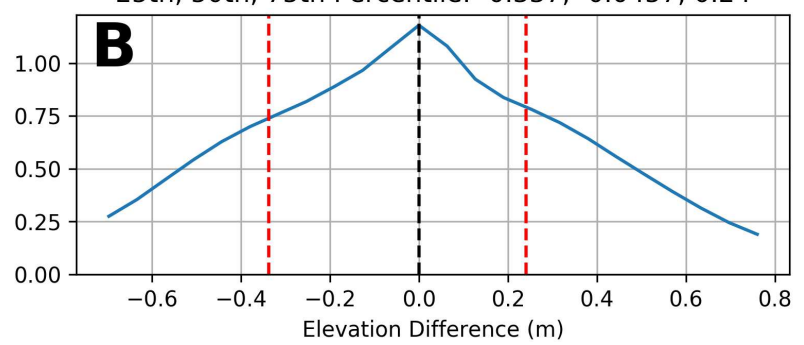

25th, 50th, 75th Percentile: $-2.72,-0.0874,2.54$

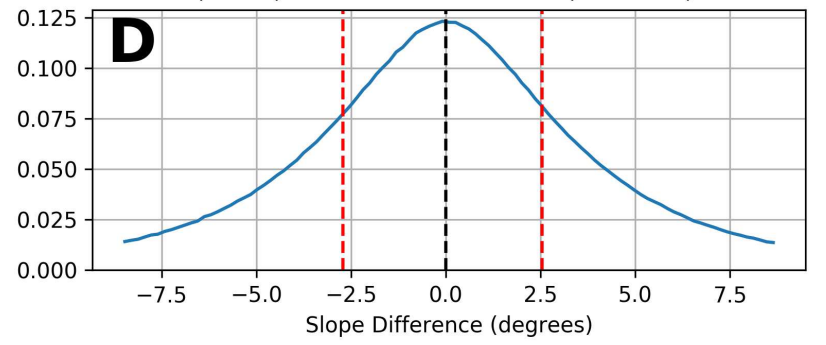

25th, 50th, 75th Percentile: $-0.31,0.0164,0.344$

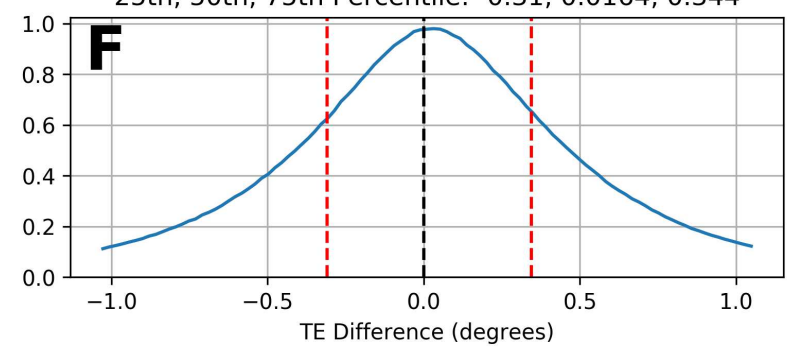

25th, 50th, 75th Percentile: $-0.252,0.00497,0.28$

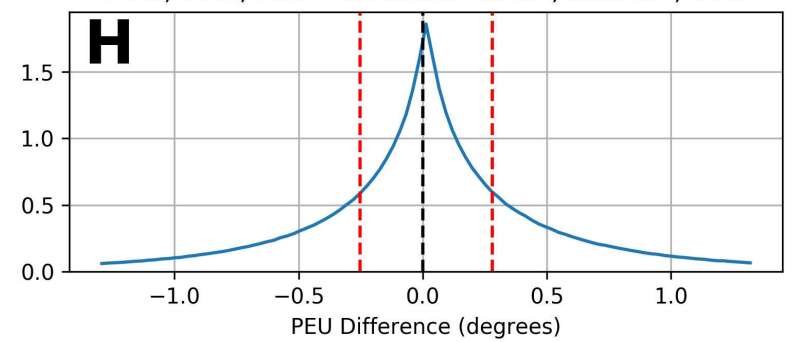

Figure S21. Pozo catchment one meter DEM difference between the LAStools TIN method LAStools (2017) and a one meter DEM generated using an inverse distance weighting (power=2, radius=grid size $\times \frac{\sqrt{2}}{2}$ ) (Points 2 Grid function (https://opentopography.org/otsoftware/points2grid), pdal.io, Shepard (1968)). Color bar scaled from 5th to 95th percentiles. Right column shows the density histogram of the differences for the whole Pozo catchment. Red lines represent the 25th and 75th percentiles, black line on zero. Histograms scaled between 5th and 95th percentiles. We refer to our github page for more detailed processing steps: https://github.com/UP-RS-ESP/TopoMetricUncertainty. 


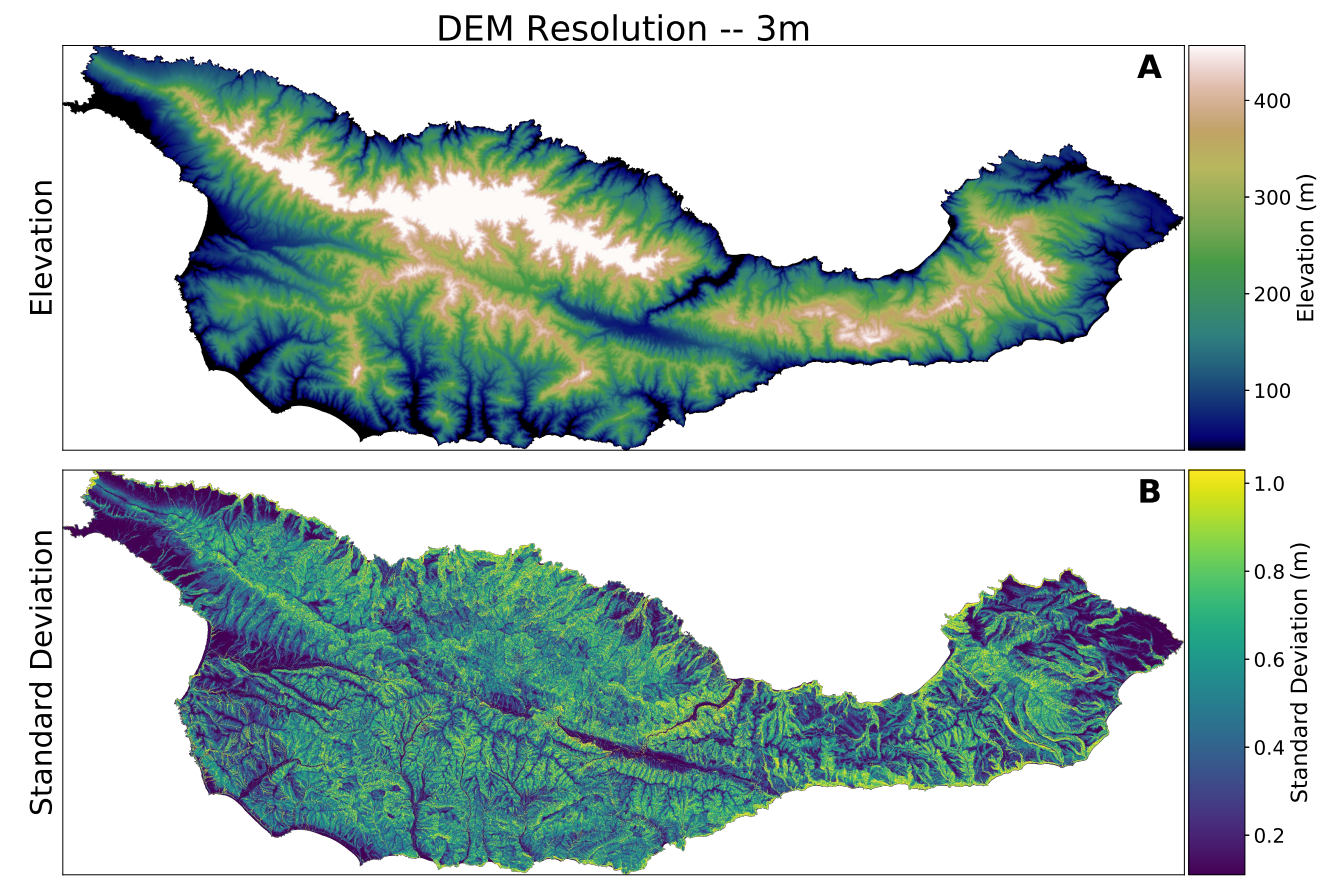

Figure S22. SCI elevation and standard deviation $-3 \mathrm{~m}$ spatial resolution. 

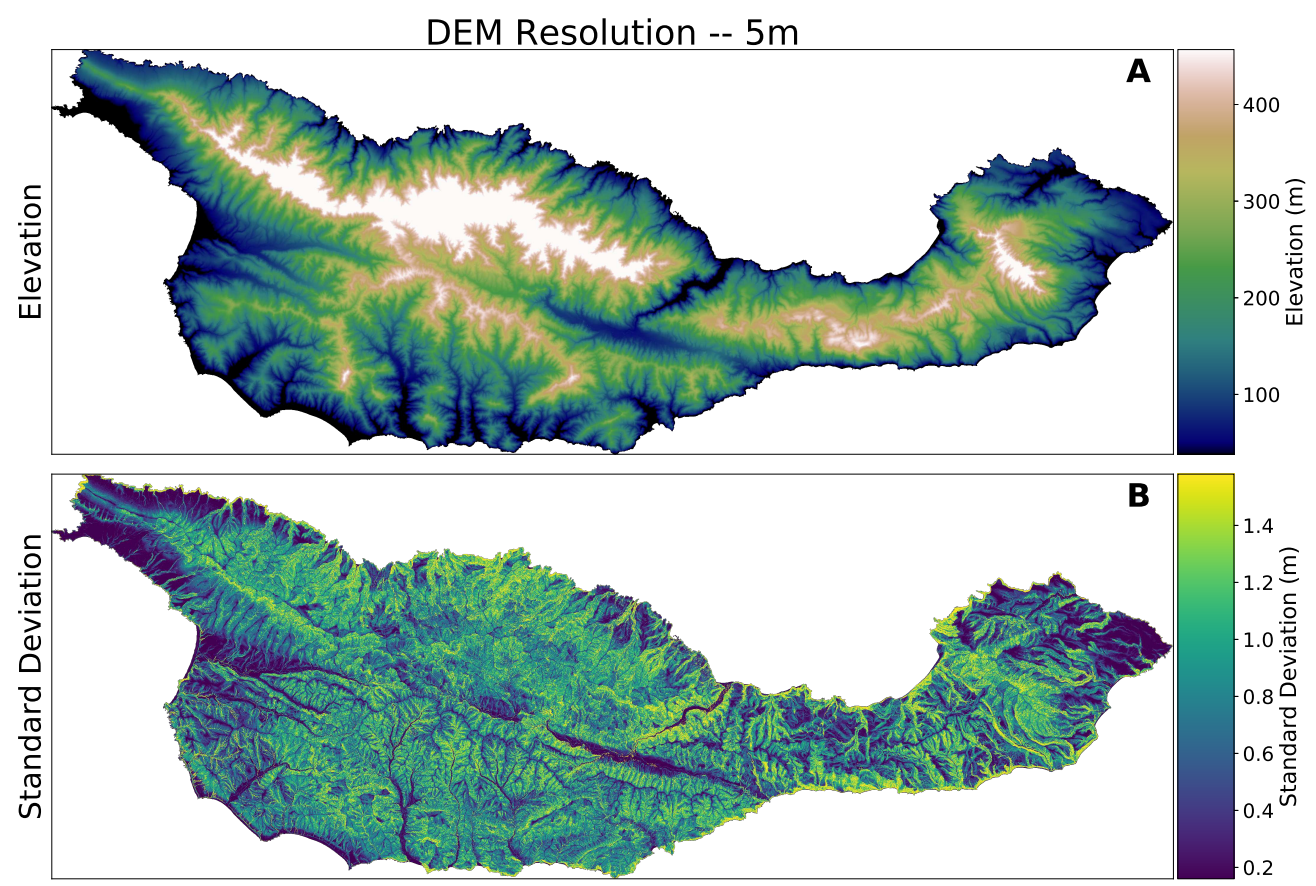

Figure S23. SCI elevation and standard deviation $-5 \mathrm{~m}$ spatial resolution.
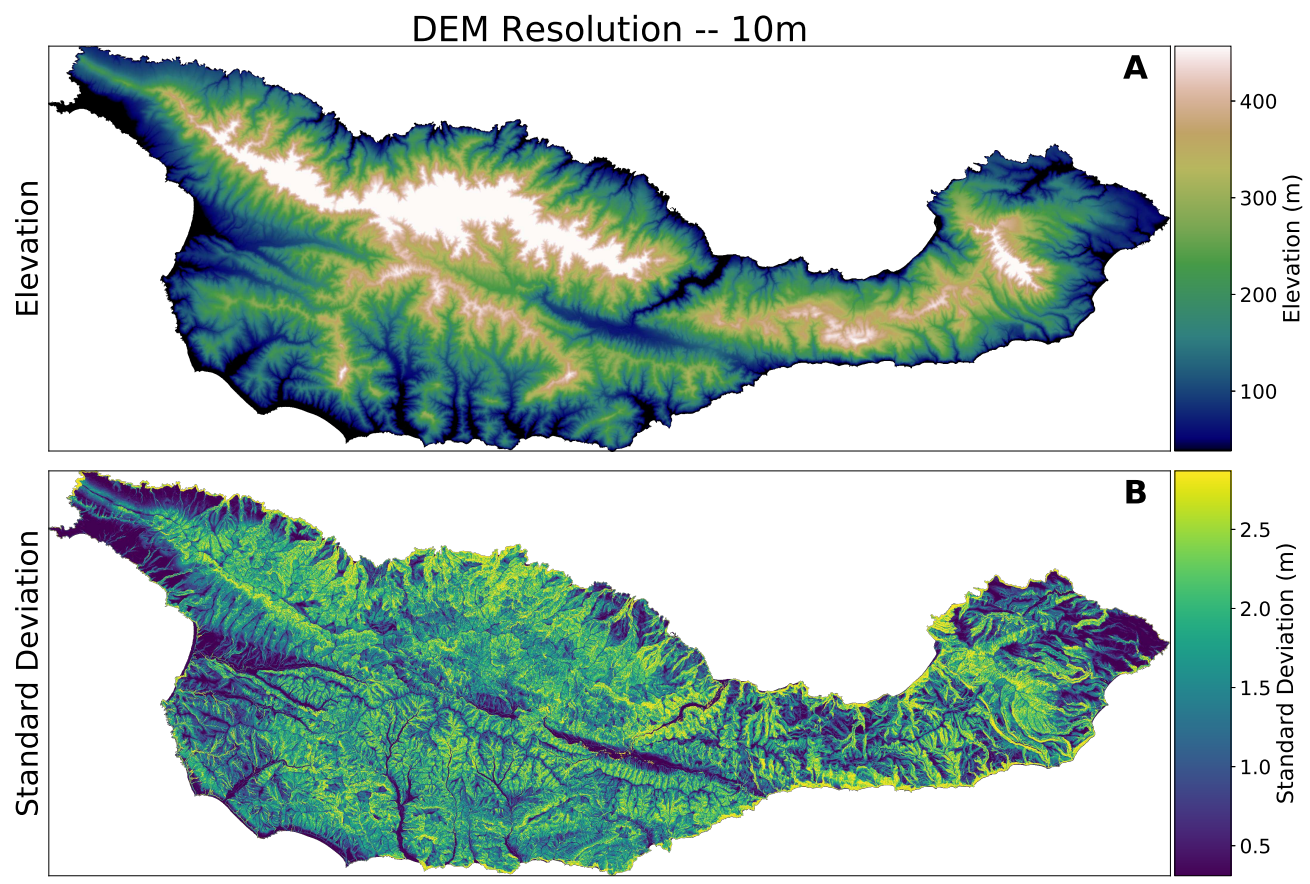

Figure S24. SCI elevation and standard deviation $-10 \mathrm{~m}$ spatial resolution. 


\subsection{Mapping Uncertainty on SCI}
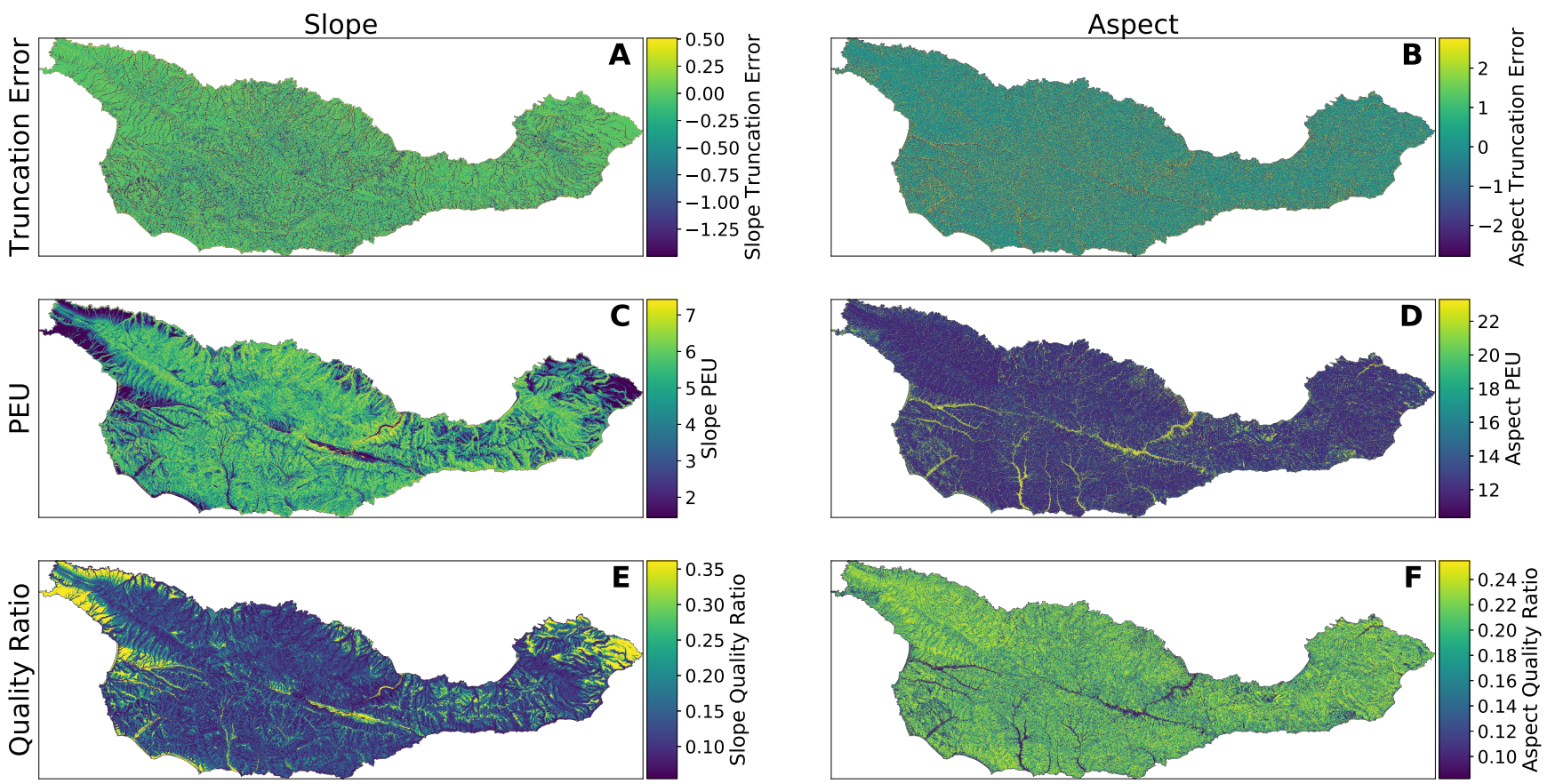

Figure S25. Truncation error (top), PEU (middle), and QR (bottom) for slope (left) and aspect (right) across the entire SCI, calculated from $5 \mathrm{~m}$ data. Both truncation and PEU are higher for aspect, leading to much lower QRs.
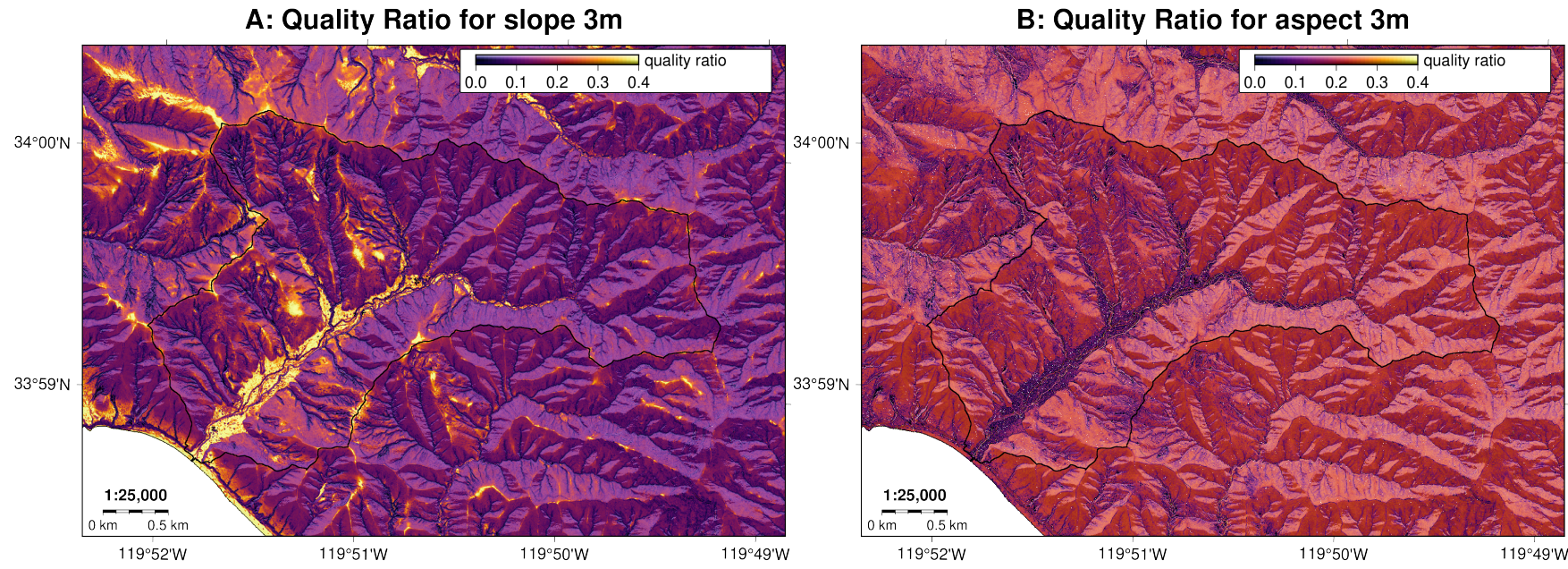

Figure S26. Slope (A, left) and aspect (B, right) QRs for the Pozo catchment (see Figure 9 for location). 

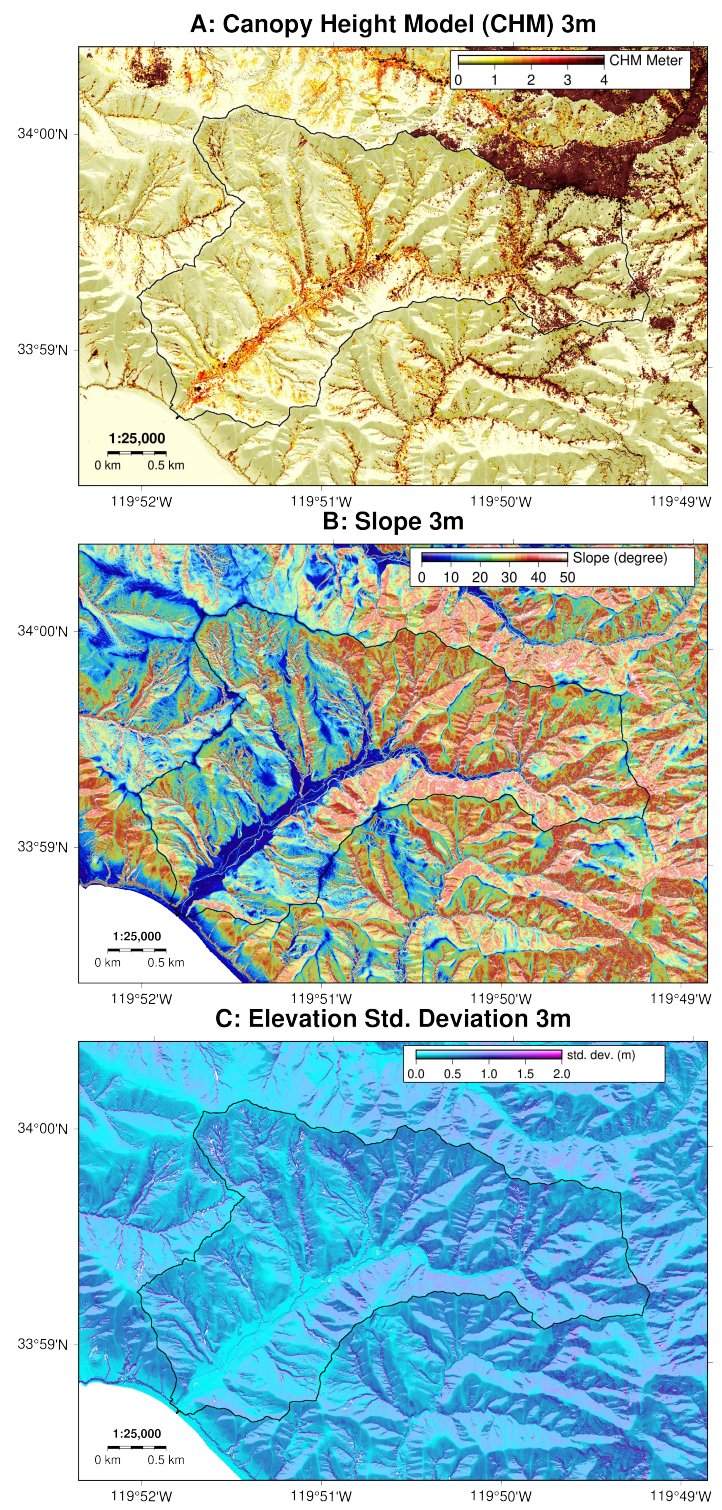

Figure S27. (A) Canopy height, (B) slope, and (C), elevation STD for the Pozo catchment (see Figure 10 for location). Standard deviation is more influenced by terrain slope than vegetation height. 


\subsection{Identification of Optimal Grid Resolution}
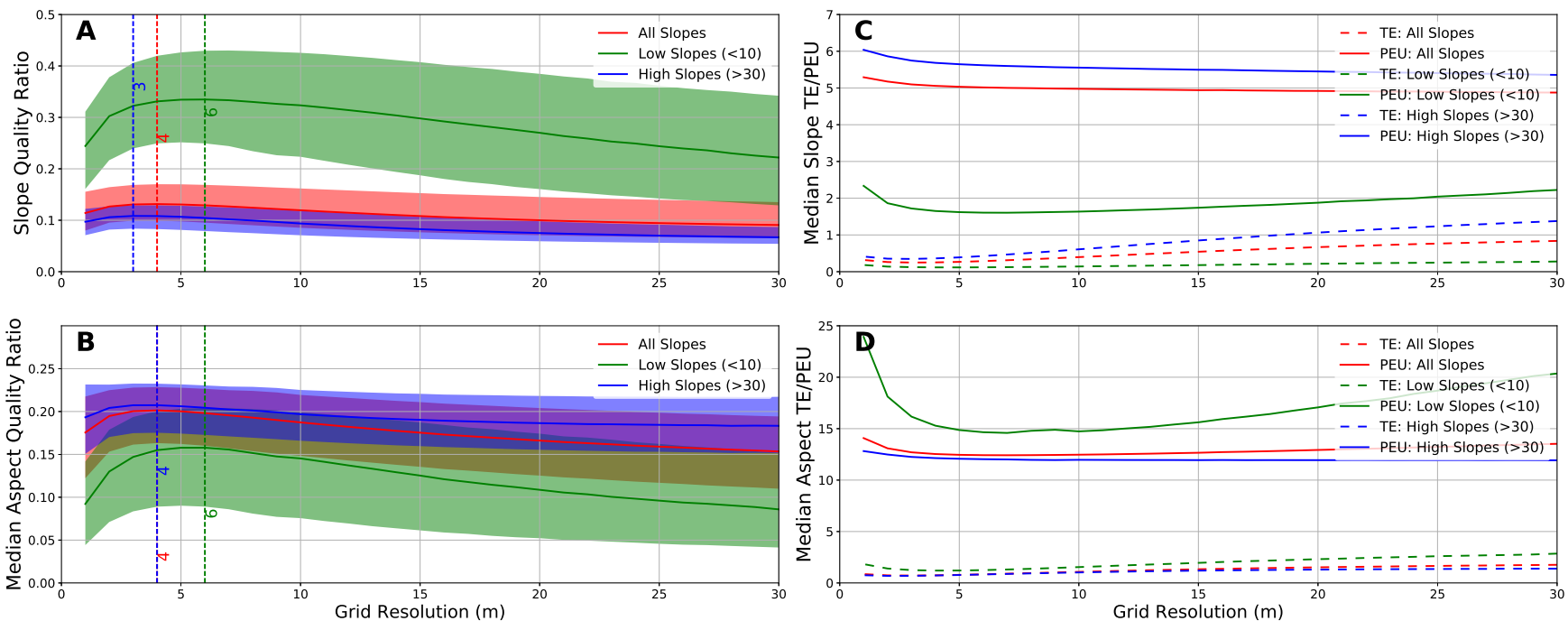

Figure S28. Median Quality Ratios (QRs) for all slopes, low slopes $(<10)$ and high slopes $(>30)$, with inter-quartile range shading. There is a clear slope bias where low slopes have better slope QRs, but worse aspect QRs. The optimal grid resolution for SCI is $4 \mathrm{~m}$ for both slope and aspect.

Table S2. Whole Island Uncertainty, TE, PEU, and QR statistics for each grid resolution.

\begin{tabular}{|l|llllllllll|}
\hline Grid Spacing & $1 \mathrm{~m}$ & $2 \mathrm{~m}$ & $3 \mathrm{~m}$ & $4 \mathrm{~m}$ & $5 \mathrm{~m}$ & $6 \mathrm{~m}$ & $7 \mathrm{~m}$ & $8 \mathrm{~m}$ & $9 \mathrm{~m}$ & $10 \mathrm{~m}$ \\
\hline Median Elevation STD & 0.16 & 0.33 & 0.49 & 0.64 & 0.8 & 0.96 & 1.11 & 1.26 & 1.41 & 1.57 \\
\hline Median Slope TE & 0.319 & 0.263 & 0.249 & 0.253 & 0.267 & 0.288 & 0.312 & 0.338 & 0.366 & 0.397 \\
Median Slope PEU & 5.29 & 5.17 & 5.1 & 5.06 & 5.03 & 5.02 & 5 & 4.99 & 4.98 & 4.98 \\
Median Slope QR & 0.114 & 0.127 & 0.131 & 0.131 & 0.131 & 0.129 & 0.127 & 0.124 & 0.122 & 0.119 \\
\hline Median Aspect TE & 0.862 & 0.74 & 0.72 & 0.743 & 0.787 & 0.841 & 0.901 & 0.961 & 1.02 & 1.08 \\
Median Aspect PEU & 14.1 & 13.1 & 12.7 & 12.5 & 12.5 & 12.4 & 12.4 & 12.4 & 12.4 & 12.5 \\
Median Aspect QR & 0.176 & 0.195 & 0.2 & 0.201 & 0.2 & 0.198 & 0.196 & 0.193 & 0.19 & 0.187 \\
\hline
\end{tabular}




\section{DEM 5m and ideal grid size for slope}

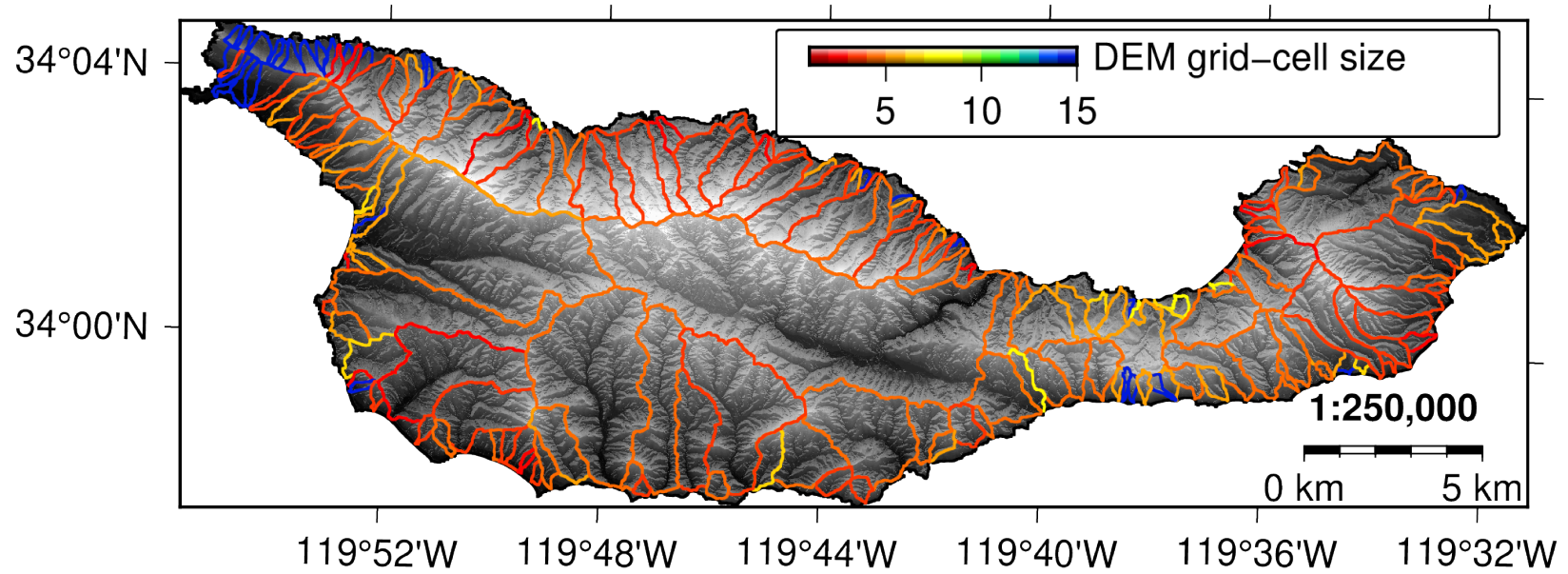

Figure S29. Optimal grid resolution for slope calculations across SCI. Most catchments have optimal resolutions around 2-5m, although some - particularly one region in the north west - have optimal spacings of more than $15 \mathrm{~m}$.

\subsection{Bias in Real-World Slope and Aspect Distributions}
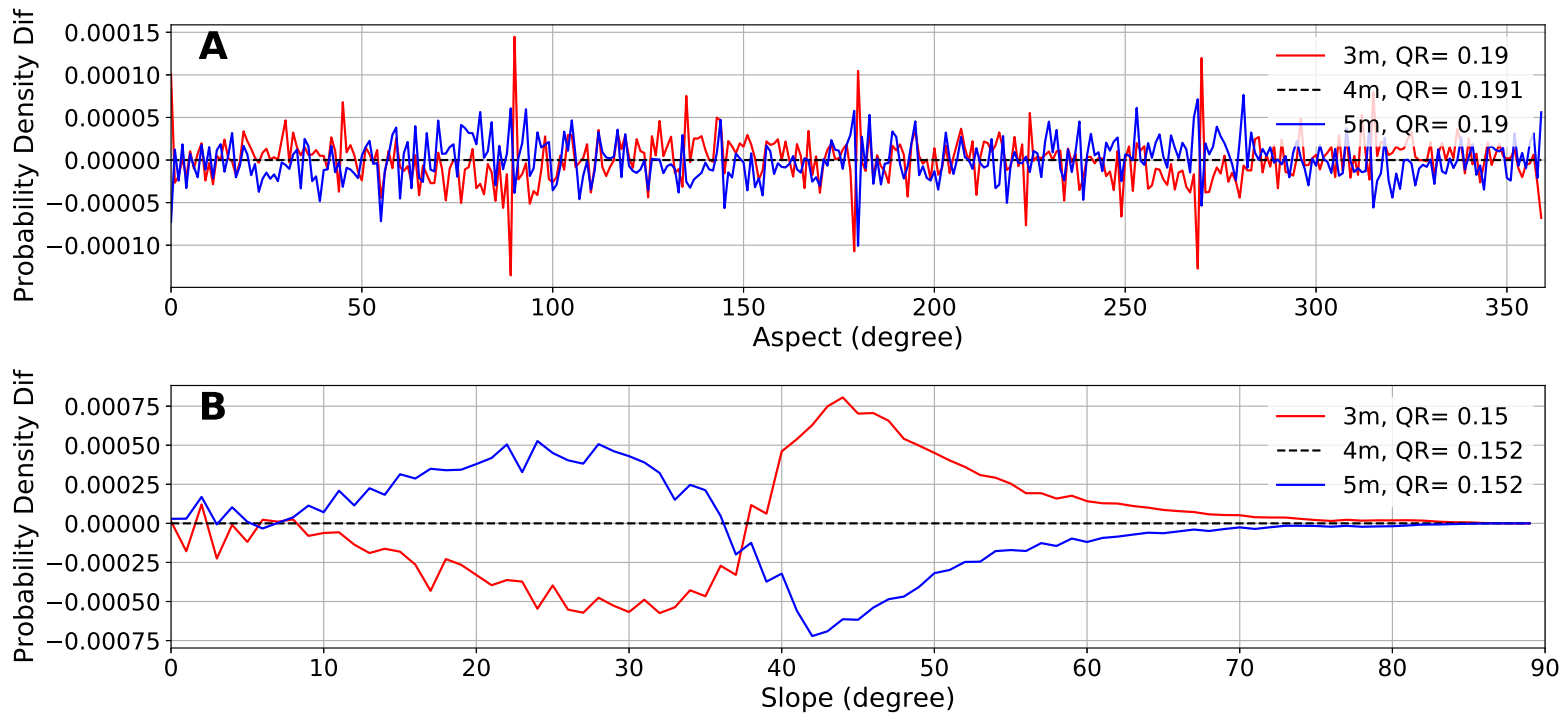

Figure S30. Divergence of high- and low- resolution slope and aspect estimates from the optimal resolution. While the differences in distribution are small, they show a distinct pattern where high slopes are overestimated on high-resolution data, and low slopes are underestimated. 

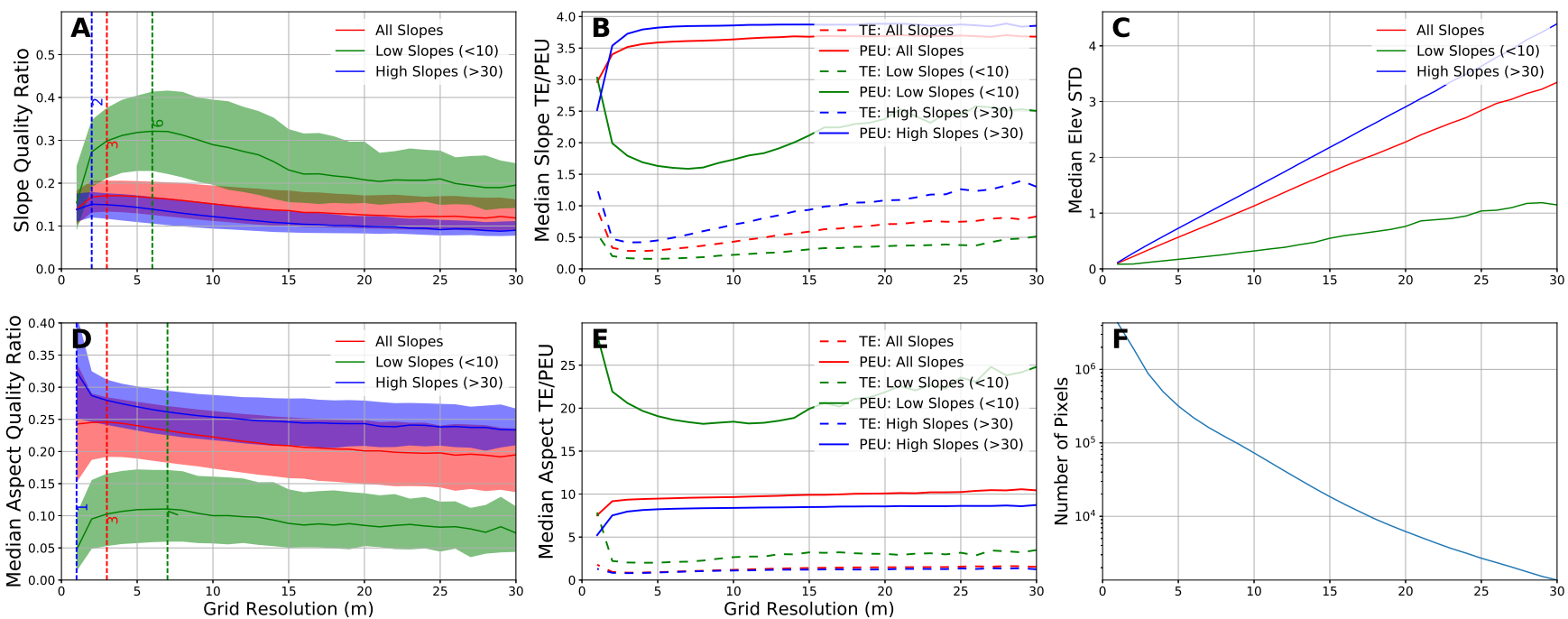

Figure S31. QR for all slopes (red), low slopes ( $<10$, green) and high slopes ( $>30$, blue) for the Pozo catchment (see Figure 10 for location). 


\section{References}

LAStools: Efficient LiDAR Processing Software (version 180831, academic), http://rapidlasso.com/LAStools, 2017.

Shepard, D.: A two-dimensional interpolation function for irregularly-spaced data, in: Proceedings of the $196823 \mathrm{rd}$ ACM national conference, pp. 517-524, ACM, 1968.

5 Watson, D.: Automatic contouring of raw data, computers and geosciences, Pergamon Press Ltd, 1, 97-101, 1982.

Wessel, P. and Luis, J. F.: The GMT/MATLAB Toolbox, Geochemistry, Geophysics, Geosystems, 18, 811-823, 2017. 